\title{
MINORS AND RESOLUTIONS OF NON-COMMUTATIVE SCHEMES
}

\author{
IGOR BURBAN, YURIY DROZD, AND VOLODYMYR GAVRAN
}

\begin{abstract}
In this article we develop the theory of minors of non-commutative schemes. This study is motivated by applications in the theory of non-commutative resolutions of singularities of commutative schemes. In particular, we construct a categorical resolution for non-commutative curves and in the rational case show that it can be realized as the derived category of a quasi-hereditary algebra.
\end{abstract}

\section{INTRODUCTION}

Let $B$ be a ring and $P$ be a finitely generated projective left $B$-module. We call the ring $A=B_{P}=\left(\operatorname{End}_{B} P\right)^{\text {op }}$ a minor of $B$. It turns out that the module categories of $B$ and $A$ are closely related.

(1) The functors $\mathrm{F}=P \otimes_{A}$ - and $\mathrm{H}=\operatorname{Hom}_{A}\left(P^{\vee},{ }_{-}\right)$from $A$-Mod to $B$-Mod are fully faithful, where $P^{\vee}=\operatorname{Hom}_{B}(P, B)$. In other words, $A$-Mod can be realized in two different ways as a full subcategory of $B$-Mod, see Theorem 4.3 .

(2) The functor $\mathrm{G}=\operatorname{Hom}_{B}\left(P,_{-}\right): B$-Mod $\longrightarrow A$-Mod is exact and essentially surjective. Moreover, we have adjoint pairs $(F, G)$ and $(G, H)$. In other words, $\mathrm{G}$ is a bilocalization functor. If

$$
I=I_{P}=\operatorname{Im}\left(P \otimes_{A} P^{\vee} \longrightarrow B\right)
$$

and $\bar{B}=B / I$ then the category $\bar{B}$-Mod is the kernel of $\mathrm{G}$ and $A$-Mod is equivalent to the Serre quotient of $B$-Mod modulo $\bar{B}$-Mod, see Theorem $4.3,2$.

(3) Under certain additional assumptions one can show that the global dimension of $B$ is finite provided the global dimensions of $A$ and $\bar{B}$ are finite, see Lemma 5.1 .

The described picture becomes even better when we pass to the (unbounded) derived categories $\mathscr{D}(A$-Mod $), \mathscr{D}(B$-Mod) and $\mathscr{D}(\bar{B}$-Mod) of the rings $A, B$ and $\bar{B}$ introduced above. Let DG be the derived functor of G, LF be the left derived functor of $\mathrm{F}$ and $\mathrm{RH}$ be the right derived functor of $\mathrm{H}$.

(1) Then we have adjoint pairs (LF,DG) and (DG, RH), the functors LF and $\mathrm{RH}$ are fully faithful and the category $\mathscr{D}(A-\mathrm{Mod})$ is equivalent to the Verdier localization of $\mathscr{D}(B$-Mod) modulo its triangulated subcategory

1991 Mathematics Subject Classification. 14F05, 14A22.

Key words and phrases. Derived categories and bilocalization and non-commutative schemes and minors. 
$\mathscr{D}_{\bar{B}}(B$-Mod $)$ consisting of complexes with cohomologies from $\bar{B}$-Mod, see Theorem 4.5

(2) Moreover, we have a semi-orthogonal decomposition

$$
\mathscr{D}(B \text {-Mod })=\left\langle\mathscr{D}_{\bar{B}}(B \text {-Mod }), \mathscr{D}(A-M o d)\right\rangle,
$$

see Corollary 2.6 .

One motivation to deal with minors comes from the theory of non-commutative crepant resolutions. Let $A$ be a commutative normal Gorenstein domain and $F$ be a reflexive $A$-module such that the ring

$$
B=B_{F}:=\operatorname{End}_{A}(A \oplus F)^{\mathrm{op}}=\left(\begin{array}{cc}
A & F \\
F^{\vee} & E
\end{array}\right),
$$

where $E=\left(\operatorname{End}_{A} F\right)^{\mathrm{op}}$, is maximal Cohen-Macaulay over $A$ and of finite global dimension. Van den Bergh suggested to view $B$ as a non-commutative crepant resolution of $A$ showing that under some additional assumptions, the existence of a non-commutative crepant resolution implies the existence of a commutative one (Van den Bergh, 2004). If we take the idempotent $e=\left(\begin{array}{ll}1 & 0 \\ 0 & 0\end{array}\right) \in B$ and pose $P=B e$ then it is easy to see that $A=B_{P}$. Thus, dealing with non-commutative (crepant) resolutions of singularities, we naturally come into the framework of the theory of minors.

In (Drozd and Greuel, 2001) it was observed that there is a close relation between coherent sheaves over the nodal cubic $C=V\left(z y^{2}-x^{3}-x^{2} z\right) \subset \mathbb{P}^{2}$ and representations of the finite dimensional algebra $\Lambda$ given by the quiver with relations

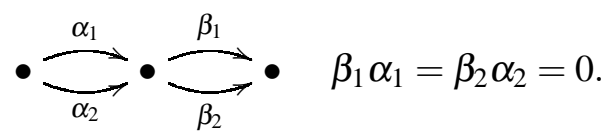

An explanation of this fact was given in (Burban and Drozd, 2011). Let $\mathscr{I}$ be the ideal sheaf of the singular point of $C$ and $\mathscr{A}=\mathscr{E} n d_{C}(\mathscr{O} \oplus \mathscr{I})$. Consider the ringed space $(C, \mathscr{A})$ and the category $\mathscr{A}$-mod of coherent left $\mathscr{A}$-modules on $C$. The the derived category $\mathscr{D}^{b}(\mathscr{A}$-mod) has a tilting complex, whose (opposite) endomorphism algebra is isomorphic to $\Lambda$ what implies that the categories $\mathscr{D}^{b}(\mathscr{A}$-mod) and $\mathscr{D}^{b}(\Lambda$-mod $)$ are equivalent. On the other hand, the triangulated category $\operatorname{Perf}(C)$ of perfect complexes on $C$ is equivalent to a full subcategory of $\mathscr{D}^{b}(\mathscr{A}$-mod $)$. In fact, we deal here with a sheaf-theoretic version of the construction of minors: the commutative scheme $(C, \mathscr{O})$ is a minor of the non-commutative scheme $(C, \mathscr{A})$. The goal of this article is to establish a general framework for the theory of minors of non-commutative schemes.

In Section 2 we review some key results on localizations of abelian and triangulated categories used in this article. In Section 3 we discuss the theory of noncommutative schemes, elaborating in particular a proof of the result characterizing the triangulated category $\operatorname{Perf}(\mathscr{A})$ of perfect complexes over a non-commutative scheme $(X, \mathscr{A})$ as the category of compact objects of the unbounded derived category of quasi-coherent sheaves $\mathscr{D}(\mathscr{A})$ (Theorem 3.14 ). Section 4 is devoted to the definition of a minor $(X, \mathscr{A})$ of a non-commutative scheme $(X, \mathscr{B})$ and the 
study of relations between $(X, \mathscr{A})$ and $(X, \mathscr{B})$. In Section 5 we introduce the notion of quasi-hereditary non-commutative schemes, which generalizes the notions of quasi-hereditary semiprimary rings (Cline et al., 1988; Dlab and Ringel, 1989) and quasi-hereditary orders (König, 1990) and study their properties. In Section 6 we elaborate the theory of strongly Gorenstein non-commutative schemes. Section 7 deals with non-commutative curves. In particular, we study here hereditary non-commutative curves. In the final Section 8, as an application of the elaborated technique, we construct a categorical resolution for any (reduced) non-commutative curve (Theorem 8.2). We call it König resolution, since it is an analogue of the construction proposed by König (König, 1991). If this curve is rational, we construct a tilting complex, which shows that this categorical resolution can be realized as the derived category of modules over a finite dimensional quasi-hereditary algebra (Theorem 8.5). In particular, it gives an estimate of the Rouquier dimension of the perfect derived category of coherent sheaves over a non-commutative curve (Corollary 8.6). For "usual" (commutative) curves this result is contained in (Burban et al., 2016).

\section{BiLOCALIZATIONS}

Recall that a full subcategory $\mathscr{C}$ of an abelian category $\mathscr{A}$ is said to be thick (or Serre subcategory) if, for any exact sequence $0 \rightarrow C^{\prime} \rightarrow C \rightarrow C^{\prime \prime} \rightarrow 0$, the object $C$ belongs to $\mathscr{C}$ if and only if both $C^{\prime}$ and $C^{\prime \prime}$ belong to $\mathscr{C}$. Then the quotient category $\mathscr{A} / \mathscr{C}$ is defined and we denote by $\Pi_{\mathscr{C}}$ the natural functor $\mathscr{A} \rightarrow \mathscr{A} / \mathscr{C}$. It is exact, essentially surjective and $\operatorname{Ker} \Pi_{\mathscr{C}}=\mathscr{C}$. For instance, if $\mathrm{G}: \mathscr{A} \rightarrow \mathscr{B}$ is an exact functor among abelian categories, its kernel $\operatorname{Ker} G$ is a thick subcategory of $\mathscr{A}$ and $\mathrm{G}$ factors as $\overline{\mathrm{G}} \cdot \Pi_{\mathrm{Ker} G}$, where $\overline{\mathrm{G}}: \mathscr{A} / \operatorname{Ker} \mathrm{G} \rightarrow \mathrm{B}$.

Analogously, if $\mathscr{C}$ is a full subcategory of a triangulated category $\mathscr{A}$, it is said to be thick if it is triangulated (i.e. closed under shifts and cones) and closed under taking direct summands. Then the quotient triangulated category $\mathscr{A} / \mathscr{C}$ is defined and we denote by $\Pi_{\mathscr{C}}$ the natural functor $\mathscr{A} \rightarrow \mathscr{A} / \mathscr{C}$. It is exact (triangulated), essentially surjective and $\operatorname{Ker} \Pi_{\mathscr{C}}=\mathscr{C}$. For instance, if $G: \mathscr{A} \rightarrow \mathscr{B}$ is an exact (triangulated) functor among triangulated categories, its kernel $\operatorname{Ker} G$ is a thick subcategory of $\mathscr{A}$ and $\mathrm{G}$ factors as $\overline{\mathrm{G}} \cdot \Pi_{\mathrm{Ker} G}$, where $\overline{\mathrm{G}}: \mathscr{A} / \mathrm{Ker} \mathrm{G} \rightarrow \mathrm{B}$.

If $\mathrm{F}: \mathscr{A} \rightarrow \mathscr{B}$ is a functor, we denote by ImF its essential image, i.e. the full subcategory of $\mathscr{B}$ consisting of objects $B$ such that there is an isomorphism $B \simeq \mathrm{FA}$ for some $A \in \mathscr{A}$. We usually use this term when $\mathrm{F}$ is a full embedding (i.e. is fully faithful), so $\operatorname{Im} F \simeq \mathscr{A}$.

We use the following well-known facts related to these notions.

Theorem 2.1. (1) Let $\mathscr{A}, \mathscr{B}$ be abelian categories, $\mathrm{G}: \mathscr{A} \rightarrow \mathscr{B}$ be an exact functor which has a left adjoint (right adjoint) $\mathrm{F}: \mathscr{B} \rightarrow \mathscr{A}$ such that the natural morphism $\mathbb{1}_{\mathscr{B}} \rightarrow \mathrm{G} \circ \mathrm{F}$ (respectively, $\mathrm{G} \circ \mathrm{F} \rightarrow \mathbb{1}_{\mathscr{B}}$ ) is an isomorphism. Let $\mathscr{C}=\operatorname{Ker} \mathrm{G}$.

(a) $\mathrm{G}=\overline{\mathrm{G}} \cdot \Pi_{\mathscr{C}}$, where $\overline{\mathrm{G}}$ is an equivalence $\mathscr{A} / \mathscr{C} \stackrel{\sim}{\rightarrow} \mathscr{B}$ and its quasiinverse functor is $\overline{\mathrm{F}}=\Pi_{\mathscr{C}}{ }^{\circ} \mathrm{F}$. 
(b) $\mathrm{F}$ is a full embedding and its essential image $\operatorname{Im} \mathrm{F}$ coincides with the left (respectively, right) orthogonal subcategory of $\mathscr{C}$, i.e. the full subcategory

${ }^{\perp_{\mathscr{C}}}=\left\{A \in \mathrm{Ob} \mathscr{A} \mid \operatorname{Hom}(A, C)=\operatorname{Ext}^{1}(A, C)=0\right.$ for all $\left.C \in \mathrm{Ob} \mathscr{C}\right\}$ (respectively,

$\mathscr{C}^{\perp}=\left\{A \in \mathrm{Ob} \mathscr{A} \mid \operatorname{Hom}(C, A)=\operatorname{Ext}^{1}(C, A)=0\right.$ for all $\left.\left.C \in \mathrm{Ob} \mathscr{C}\right\}.\right)$

(c) $\mathscr{C}=\left({ }^{\perp} \mathscr{C}\right)^{\perp}$ (respectively, $\mathscr{C}={ }^{\perp}\left(\mathscr{C}^{\perp}\right)$ ).

(d) The embedding functor $\mathscr{C} \rightarrow \mathscr{A}$ has a left (respectively, right) adjoint.

(2) Let $\mathscr{A}, \mathscr{B}$ be triangulated categories, G: $\mathscr{A} \rightarrow \mathscr{B}$ be an exact (triangulated) functor which has a left adjoint (right adjoint) $\mathrm{F}: \mathscr{B} \rightarrow \mathscr{A}$ such that the natural morphism $\mathbb{1}_{\mathscr{B}} \rightarrow \mathrm{G} \circ \mathrm{F}$ (respectively, $\mathrm{G} \circ \mathrm{F} \rightarrow \mathbb{1}_{\mathscr{B}}$ ) is an isomorphism. Let $\mathscr{C}=\operatorname{Ker} \mathrm{G}$.

(a) $\mathrm{G}=\overline{\mathrm{G}} \cdot \Pi_{\mathscr{C}}$, where $\overline{\mathrm{G}}$ is an equivalence $\mathscr{A} / \mathscr{C} \stackrel{\sim}{\rightarrow} \mathscr{B}$ and its quasiinverse functor is $\overline{\mathrm{F}}=\Pi_{\mathscr{C}}{ }^{\circ} \mathrm{F}$.

(b) $\mathrm{F}$ is a full embedding and its essential image Im F coincides with the left (respectively, right) orthogonal subcategory of $\mathscr{C}$, i.e. the full subcategory 1

$$
{ }^{\perp} \mathscr{C}=\{A \in \mathrm{Ob} \mathscr{A} \mid \operatorname{Hom}(A, C)=0 \text { for all } C \in \mathrm{Ob} \mathscr{C}\}
$$

(respectively,

$$
\left.\mathscr{C}^{\perp}=\{A \in \mathrm{Ob} \mathscr{A} \mid \operatorname{Hom}(C, A)=0 \text { for all } C \in \operatorname{Ob} \mathscr{C}\} .\right)
$$

(c) $\mathscr{C}=\left({ }^{\perp} \mathscr{C}\right)^{\perp}$ (respectively, $\mathscr{C}={ }^{\perp}\left(\mathscr{C}^{\perp}\right)$ ).

(d) The embedding functor $\mathscr{C} \rightarrow \mathscr{A}$ has a left (respectively, right) adjoint, which induces an equivalence $\mathscr{A} /{ }^{\perp} \mathscr{C} \stackrel{\sim}{\rightarrow} \mathscr{C}$ (respectively, $\mathscr{A} / \mathscr{C}^{\perp} \stackrel{\sim}{\rightarrow}$ $\mathscr{C})$.

Proof. The statement (1a) is proved in (Gabriel, 1962, Ch. III, Proposition 5) if $F$ is right adjoint of $G$. The case of left adjoint is just a dualization. The proof of the statement (2a) is quite analogous. Therefore, from now on we can suppose that $\mathscr{B}=\mathscr{A} / \mathscr{C}$. Then the statements (1b) and (2b) are just (Gabriel, 1962, p.371, Lemma 2 et Corollaire) and (Neeman, 2001, Theorem 9.1.16). The statements (1c) and (2c) are (Geigle and Lenzing, 1991, Corollary 2.3) and (Neeman, 2001, Corollary 9.1.14). Thus the statement (2d) also follows from (Neeman, 2001, Theorem 9.1.16). In the abelian case the left (respectively, right) adjoint $\mathrm{J}$ to the embedding $\mathscr{C} \rightarrow \mathscr{A}$ is given by the rule $A \mapsto \operatorname{Cok} \Psi(A)$ (respectively, $A \mapsto \operatorname{Ker} \Psi(A)$ ), where $\Psi$ is the natural morphism $\mathrm{F} \circ \mathrm{G} \rightarrow \mathbb{1}_{\mathscr{A}}$ (respectively, $\mathbb{1}_{\mathscr{A}} \rightarrow \mathrm{F} \circ \mathrm{G}$ ).

Remark 2.2. Note that in the abelian case the composition $\Pi_{\perp \mathscr{C}} \circ \mathrm{J}$ (respectively, $\left.\Pi_{\mathscr{C} \perp}{ }^{\circ}\right)$ need not be an equivalence. The reason is that the subcategory ${ }^{\perp} \mathscr{C}\left(\mathscr{C}^{\perp}\right)$ need not be thick (see (Geigle and Lenzing, 1991)).

\footnotetext{
${ }^{1}$ Note that in the book (Neeman, 2001) the notations for the orthogonal subcategories are opposite to ours. The latter seems more usual, especially in the representation theory, see, for instance, (Alonso et al., 2000; Geigle and Lenzing, 1991). In (Gabriel, 1962) the objects of the right orthogonal subcategory $\mathscr{C}^{\perp}$ are called $\mathscr{C}$-closed.
} 
A thick subcategory $\mathscr{C}$ of an abelian or triangulated category $\mathscr{A}$ is said to be localizing (colocalizing) if the canonical functor $\mathrm{G}: \mathscr{A} \rightarrow \mathscr{A} / \mathscr{C}$ has a right (respectively, left) adjoint F. Neeman (Neeman, 2001) calls F a Bousfield localization (respectively, a Bousfield colocalization) ${ }^{2}$ In this case the natural morphism $\mathrm{G} \circ \mathrm{F} \rightarrow \mathbb{1}_{\mathscr{A} / \mathscr{C}}$ (respectively, $\mathbb{1}_{\mathscr{A} / \mathscr{C}} \rightarrow \mathrm{G} \circ \mathrm{F}$ ) is an isomorphism (Gabriel, 1962, Ch.III,Proposition 3), (Neeman, 2001, Lemma 9.1.7). If $\mathscr{C}$ is both localizing and colocalizing, we call it bilocalizing and call the category $\mathscr{A} / \mathscr{C}$ (or any equivalent one) a bilocalization of $\mathscr{A}$. We also say in this case that $\mathrm{G}$ is a bilocalization functor. In other words, an exact functor $\mathrm{G}: \mathscr{A} \rightarrow \mathscr{B}$ is a bilocalization functor if it has both left adjoint $\mathrm{F}$ and right adjoint $\mathrm{H}$ and the natural morphisms $\mathbb{1}_{\mathscr{B}} \rightarrow \mathrm{GF}$ and $\mathrm{GH} \rightarrow \mathbb{1}_{\mathscr{B}}$ are isomorphisms.

Corollary 2.3. Let $\mathrm{G}: \mathscr{A} \rightarrow \mathscr{B}$ be an exact functor between abelian or triangulated categories which has both left adjoint $\mathrm{F}$ and right adjoint $\mathrm{H}$. In order that $\mathrm{G}$ will be a bilocalization functor it is necessary and sufficient that one of the natural morphisms $\mathbb{1}_{\mathscr{B}} \rightarrow \mathrm{G} \circ \mathrm{F}$ or $\mathrm{G} \circ \mathrm{H} \rightarrow \mathbb{1}_{\mathscr{B}}$ be an isomorphism.

Proof. Let, for instance, the first of these morphisms be an isomorphism. Then there is an equivalence of categories $\overline{\mathrm{G}}: \mathscr{A} / \mathrm{KerG} \stackrel{\sim}{\rightarrow} \mathscr{B}$ such that $\mathrm{G}=\overline{\mathrm{G}} \Pi_{\mathscr{C}}$, where $\mathscr{C}=\operatorname{Ker} G$. So we can suppose that $\mathscr{B}=\mathscr{A} / \mathscr{C}$ and $\mathrm{G}=\Pi_{\mathscr{C}}$. Thus the morphism $\mathrm{GH} \rightarrow \mathbb{1}_{\mathscr{B}}$ is an isomorphism, since $\mathrm{H}$ is right adjoint to $\mathrm{G}$.

Corollary 2.4. Let $\mathscr{C}$ be a localizing (colocalizing) thick subcategory of an abelian category $\mathscr{A}, \mathscr{D}_{\mathscr{C}}(\mathscr{A})$ be the full subcategory of $\mathscr{D}(\mathscr{A})$ consisting of all complexes $C^{\bullet}$ such that all cohomologies $H^{i}\left(C^{\bullet}\right)$ are in $\mathscr{C}$. Suppose that the Bousfield localization (respectively, colocalization) functor $\mathrm{F}$ has right (respectively, left) derived functor. Then $\mathscr{D}_{\mathscr{C}}(\mathscr{A})$ is also a localizing (colocalizing) subcategory of $\mathscr{A}$ and $\mathscr{D}(\mathscr{A} / \mathscr{C}) \simeq \mathscr{D}(\mathscr{A}) / \mathscr{D}_{\mathscr{C}}(\mathscr{A})$.

Proof. We consider the case of a localizing subcategory $\mathscr{C}$, denote by $\mathrm{G}$ the canonical functor $\mathscr{A} \rightarrow \mathscr{A} / \mathscr{C}$ and by $\mathrm{F}$ its right adjoint. As $\mathrm{G}$ is exact, it induces an exact functor $\mathscr{D}(\mathscr{A}) \rightarrow \mathscr{D}(\mathscr{A} / \mathscr{C})$ acting on complexes componentwise. We denote it by DG; it is both right and left derived of G. Obviously, KerDG $=\mathscr{D}_{\mathscr{C}}(\mathscr{A})$. Since $\mathrm{G} \cdot \mathrm{F} \rightarrow \mathbb{1}_{\mathscr{A} / \mathscr{C}}$ is an isomorphism, the morphism $\mathrm{DG} \cdot \mathrm{RF} \rightarrow \mathbb{1}_{\mathscr{D}(\mathscr{A} / \mathscr{C})}$ is also an isomorphism, so we can apply Theorem 2.1(2).

Remark 2.5. (1) If $\mathscr{C}$ is localizing and $\mathscr{A}$ is a Grothendieck category, the right derived functor RF exists (Alonso et al., 2000), so $\mathscr{D}(\mathscr{A} / \mathscr{C}) \simeq \mathscr{D}_{\mathscr{C}}(\mathscr{A})$. We do not know general conditions which ensure the existence of the left derived functor LF in the case of colocalizing categories, though it exists when $\mathscr{A}$ is a category of quasi-coherent modules over a quasi-compact separated non-commutative scheme and $\mathrm{F}$ is tensor product or inverse image, see Proposition 3.12 .

(2) Miyatchi (Miyachi, 1991) proved that always $\mathscr{D}^{\sigma}(\mathscr{A} / \mathscr{C}) \simeq \mathscr{D}_{\mathscr{C}}^{\sigma}(\mathscr{A})$, where $\sigma \in\{+,-, b\}$.

\footnotetext{
${ }^{2}$ Actually, Neeman uses this term for triangulated categories, but we will use it for abelian categories too.
} 
We recall that a sequence $\left(\mathscr{A}_{1}, \mathscr{A}_{2}, \ldots, \mathscr{A}_{m}\right)$ of triangulated subcategories of a triangulated category $\mathscr{A}$ is said to be a semi-orthogonal decomposition of $\mathscr{A}$ if $\operatorname{Hom}\left(A, A^{\prime}\right)=0$ for $A \in \mathscr{A}_{i}, A^{\prime} \in \mathscr{A}_{j}$ and $i>j$, and for every object $A \in \mathscr{A}$ there is a chain of morphisms

$$
0=A_{m} \stackrel{f_{m}}{\longrightarrow} A_{m-1} \stackrel{f_{m-1}}{\longrightarrow} \ldots A_{2} \stackrel{f_{2}}{\longrightarrow} A_{1} \stackrel{f_{1}}{\rightarrow} A_{0}=A
$$

such that Cone $f_{i} \in \mathscr{A}_{i}$ (Kuznetsov and Lunts, 2012).

Corollary 2.6. Let $\mathrm{G}: \mathscr{A} \rightarrow \mathscr{B}$ be an exact functor among triangulated categories, $\mathrm{F}: \mathscr{B} \rightarrow \mathscr{A}$ be its right (left) adjoint such that the natural morphism $\phi: \mathbb{1}_{\mathscr{B}} \rightarrow \mathrm{GF}$ (respectively, $\psi: \mathrm{GF} \rightarrow \mathbb{1}_{\mathscr{B}}$ ) is an isomorphism. Then $(\mathrm{Im} \mathrm{F}, \operatorname{Ker} \mathrm{G})$ (respectively, $(\mathrm{Ker} \mathrm{G}, \mathrm{Im} \mathrm{F})$ ) is a semi-orthogonal decomposition of $\mathscr{A}$.

Proof. We consider the case of left adjoint. If $A=\mathrm{F} B$ and $A^{\prime} \in \operatorname{Ker} \mathrm{G}$, then $\operatorname{Hom}_{\mathscr{A}}\left(A, A^{\prime}\right) \simeq$ $\operatorname{Hom}_{\mathscr{B}}(\mathrm{G} A, B)=0$. On the other hand, consider the natural morphism $f: \mathrm{FG} A \rightarrow A$. Then $\mathrm{G} f$ is an isomorhism, whence Cone $f \in \operatorname{Ker}$. So we can set $A_{1}=$ FGA, $f_{1}=f$.

\section{NON-COMMUTATIVE SCHEMES}

Definition 3.1. (1) A non-commutative scheme is a pair $(X, \mathscr{A})$, where $X$ is a scheme (called the commutative background of the non-commutative scheme) and $\mathscr{A}$ is a sheaf of $\mathscr{O}_{X}$-algebras, which is quasi-coherent as a sheaf of $\mathscr{O}_{X}$-modules. Sometimes we say "non-commutative scheme $\mathscr{A}^{\text {" }}$ not mentioning its commutative background $X$. We denote by $X_{\mathrm{cl}}$ the set of closed points of $X$.

(2) A non-commutative scheme $(X, \mathscr{A})$ is said to be affine (separated, quasicompact) if so is its commutative background $X$. It is said to be reduced if $\mathscr{A}$ has no nilpotent ideals.

(3) A morphism of non-commutative schemes $f:(Y, \mathscr{B}) \rightarrow(X, \mathscr{A})$ is a pair $\left(f_{X}, f^{\#}\right)$, where $f_{X}: Y \rightarrow X$ is a morphism of schemes and $f^{\#}$ is a morphism of $f_{X}^{-1} \mathscr{O}_{X}$-algebras $f_{X}^{-1} \mathscr{A} \rightarrow \mathscr{B}$. In what follows we usually write $f$ instead of $f_{X}$.

(4) Given a non-commutative scheme $(X, \mathscr{A})$, we denote by $\mathscr{A}$-Mod (respectively, by $\mathscr{A}$-mod) the category of quasi-coherent (respectively, coherent) sheaves of $\mathscr{A}$-modules. We call objects of this category just $\mathscr{A}$-modules (respectively, coherent $\mathscr{A}$-modules).

(5) If $f:(Y, \mathscr{B}) \rightarrow(X, \mathscr{A})$ is a morphism of non-commutative schemes, we denote by $f^{*}: \mathscr{A}$-Mod $\rightarrow \mathscr{B}$-Mod the functor of inverse image which maps an $\mathscr{A}$-module $\mathscr{M}$ to the $\mathscr{B}$-module $\mathscr{B} \otimes_{f^{-1} \mathscr{A}} f^{-1} \mathscr{M}$. If the map $f_{X}$ is separated and quasi-compact, we denote by $f_{*}: \mathscr{B}$-Mod $\rightarrow \mathscr{A}$-Mod the functor of direct image. It follows from (Grothendieck, 1960, $\S 0.1$ and $\S 1.9 .2)$ that these functors are well-defined. Moreover, $f^{*}$ maps coherent modules to coherent ones.

In this paper we always suppose non-commutative schemes separated and quasicompact. 
Remark 3.2. (1) If $(X, \mathscr{A})$ is affine, i.e. $X=\operatorname{Spec} R$ for some commutative $\operatorname{ring} R$, then $\mathscr{A}=A^{\sim}$ is a sheafification of an $R$-algebra $A$. A quasi-coherent $\mathscr{A}$-module is just a sheafification $M^{\sim}$ of an $A$-module $M$, so $\mathscr{A}$-Mod $\simeq$ $A$-Mod and we identify these categories. If, moreover, $A$ is noetherian, then $\mathscr{A}$-mod coincides with the category $A$-mod of finitely generated $A$ modules.

(2) If $X$ is separated and quasi-compact, $\mathscr{A}$-Mod is a Grothendieck category. In particular, every quasi-coherent $\mathscr{A}$-module has an injective envelope. We denote by $\mathscr{A}$-Inj the full subcategory of $\mathscr{A}$-Mod consisting of injective modules.

(3) The inverse image functor $f^{*}$ for a morphism of non-commutative schemes usually does not coincide with the inverse image functor $f_{X}^{*}$ with respect to the morphism of their commutative backgrounds. We can guarantee it if $\mathscr{B}=f_{X}^{*} \mathscr{A}$, for instance, if $Y$ is an open subset of $X$ and $\mathscr{B}=\left.\mathscr{A}\right|_{Y}$.

Definition 3.3. (1) The center of $\mathscr{A}$ is the subsheaf cen $\mathscr{A} \subseteq \mathscr{A}$ such that

$(\operatorname{cen} \mathscr{A})(U)=\left\{\alpha \in \mathscr{A}(U)|\alpha|_{V} \in \operatorname{cen} \mathscr{A}(V)\right.$ for all $\left.V \subseteq U\right\}$,

where cen $A$ denotes the center of a ring $A$.

(2) We say that a non-commutative scheme $(X, \mathscr{A})$ is central, if the natural homomorphism $\mathscr{O}_{X} \rightarrow \mathscr{A}$ maps $\mathscr{O}_{X}$ bijectively onto the center cen $(\mathscr{A})$ of $\mathscr{A}$.

Note that if $(X, \mathscr{A})$ is affine, $X=\operatorname{Spec} R$ and $\mathscr{A}=A^{\sim}$, then $\operatorname{cen} \mathscr{A}=(\operatorname{cen} A)^{\sim}$.

Proposition 3.4. End $\mathbb{1}_{\mathscr{A}-\text { Mod }} \simeq \operatorname{End} \mathbb{1}_{\mathscr{A}-\mathrm{Inj}} \simeq \Gamma(X$, cen $\mathscr{A})$.

Proof. Let $\alpha \in \Gamma(X$,cen $\mathscr{A})$. Given any $\mathscr{M} \in \mathscr{A}$-Mod, define $\alpha(\mathscr{M}): \mathscr{M} \rightarrow \mathscr{M}$ by the rule: $\alpha(M)(U): \mathscr{M}(U) \rightarrow \mathscr{M}(U)$ is the multiplication by $\alpha \mid U$ for every open $U \subseteq X$. Obviously, it is a morphism of $\mathscr{A}$-modules. Moreover, if $f \in$ $\operatorname{Hom}_{\mathscr{A}}(\mathscr{M}, \mathscr{N})$, one easily sees that $f \alpha(M)=\alpha(N) f$, so $\alpha$ defines an element from End $\mathbb{1}_{\mathscr{A} \text {-Mod. }}$

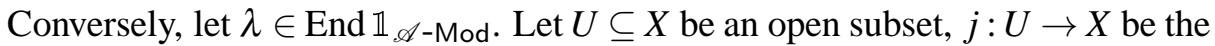
embedding. Then $\lambda(U)=\lambda\left(j_{*} j^{*} \mathscr{A}\right)$ is an element from $\operatorname{End}_{\mathscr{A}}\left(j_{*} j^{*} \mathscr{A}\right)=\mathscr{A}(U)$. Since $\lambda$ is an endomorphism of the identity functor, $\lambda(U)$ it is in cen $\mathscr{A}(U)$. Moreover, if $V \subseteq U$ is another open subset, $j^{\prime}: V \rightarrow X$ is the embedding, the restriction homomorphism $r: j_{*} j^{*} \mathscr{A} \rightarrow j_{*}^{\prime} j^{\prime *} \mathscr{A}$ gives the commutative diagram

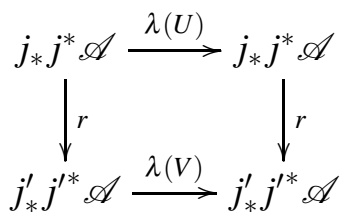

It implies that $\lambda(V)=\lambda(U) \mid V$. In particular, $\lambda(X)=\alpha$ is an element from $\Gamma(X$, cen $\mathscr{A})$ and $\lambda(U)$ coincides with the multiplication by $\alpha \mid U$. Thus we obtain an isomorphism End $\mathbb{1}_{\mathscr{A} \text {-Mod }} \simeq \Gamma(X$, cen $\mathscr{A})$.

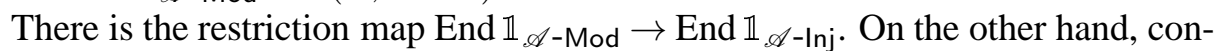
sider an injective copresentation of an $\mathscr{A}$-module $\mathscr{M}$, i.e. an exact sequence $0 \rightarrow$ 
$\mathscr{M} \stackrel{\alpha_{\mathscr{M}}}{\rightarrow} \mathscr{I}_{\mathscr{M}} \rightarrow \mathscr{I}_{\mathscr{M}}^{\prime}$ with injective modules $\mathscr{I}_{\mathscr{M}}$ and $\mathscr{I}_{\mathscr{M}}^{\prime}$. Let $\lambda \in$ End $\mathbb{1}_{\mathscr{A}}$-Inj. Then there is a unique homomorphism $\lambda(\mathscr{M}): \mathscr{M} \rightarrow \mathscr{M}$ such that $\lambda\left(\mathscr{I}_{\mathscr{M}}\right) \alpha_{\mathscr{M}}=$ $\alpha_{\mathscr{M}} \lambda(\mathscr{M})$. Let $0 \rightarrow \mathscr{N}^{\alpha_{\mathscr{K}}} \mathscr{I}_{\mathscr{N}} \rightarrow \mathscr{I}_{\mathscr{N}}^{\prime}$ be an injective copresentation of another $\mathscr{A}$-module $\mathscr{N}$ and $f \in \operatorname{Hom}_{\mathscr{A}}(\mathscr{M}, \mathscr{N})$. Extending $f$ to injective copresentations, we obtain a commutative diagram

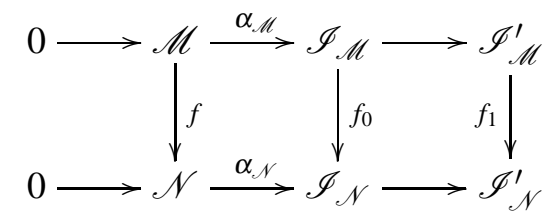

It implies that

$$
\begin{aligned}
\alpha_{\mathscr{N}} \lambda(\mathscr{N}) f=\lambda\left(\mathscr{I}_{\mathscr{N}}\right) \alpha_{\mathscr{N}} f & =\lambda\left(\mathscr{I}_{\mathscr{N}}\right) f_{0} \alpha_{\mathscr{M}} \\
& =f_{0} \lambda\left(\mathscr{I}_{\mathscr{M}}\right) \alpha_{\mathscr{M}}=f_{0} \alpha_{\mathscr{M}} \lambda(\mathscr{M})=\alpha_{\mathscr{N}} f \lambda(\mathscr{M}),
\end{aligned}
$$

whence $\lambda(\mathscr{N}) f=f \lambda(\mathscr{M})$, so we have extended $\lambda$ to a unique endomorphism of $\mathbb{1}_{\mathscr{A} \text {-Mod. }}$

Proposition 3.5. Let $\mathscr{C}=\operatorname{cen}(\mathscr{A}), X^{\prime}=\operatorname{Spec} \mathscr{C}$ be the spectrum of the (commutative) $\mathscr{O}_{X}$-algebra $\mathscr{C}, \phi: X^{\prime} \rightarrow X$ be the structural morphism, and $\mathscr{A}^{\prime}=\phi^{-1} \mathscr{A}$.

(1) $\mathscr{A}^{\prime}$ is an $\mathscr{O}_{X^{\prime}}$-algebra, so $\left(X^{\prime}, \mathscr{A}^{\prime}\right)$ is a central non-commutative scheme.

(2) For any $\mathscr{F} \in \mathscr{A}$-Mod the natural map $\mathscr{F} \rightarrow \phi_{*} \phi^{*} \mathscr{F}$ is an isomorphism 3

(3) For any $\mathscr{F}^{\prime} \in \mathscr{A}^{\prime}$-Mod the natural map $\phi^{*} \phi_{*} \mathscr{F}^{\prime} \rightarrow \mathscr{F}^{\prime}$ is an isomorphism.

(4) The functors $\phi^{*}$ and $\phi_{*}$ establish an equivalence of the categories $\mathscr{A}-\mathrm{Mod}$ and $\mathscr{A}^{\prime}$-Mod as well as of $\mathscr{A}$-mod and $\mathscr{A}^{\prime}$-mod.

Thus, when necessary, we can suppose, without loss of generality, that our noncommutative schemes are central.

Proof. All claims are obviously local, so we can suppose that $X=\operatorname{Spec} R$ and $X^{\prime}=\operatorname{Spec} R^{\prime}$, where $R^{\prime}$ is the center of the $R$-algebra $A=\Gamma(X, \mathscr{A})$. Then all claims are trivial.

We call a non-commutative scheme $(X, \mathscr{A})$ noetherian if the scheme $X$ is noetherian and $\mathscr{A}$ is coherent as a sheaf of $\mathscr{O}_{X}$-modules. Note that if $(X, \mathscr{A})$ is noetherian, the central non-commutative scheme $\left(X^{\prime}, \mathscr{A}^{\prime}\right)$ constructed in Proposition 3.5 is also noetherian. Indeed, if an affine non-commutative scheme $\left(\operatorname{Spec} R, A^{\sim}\right)$ is noetherian, then $A$ is a noetherian algebra, i.e. $C=\operatorname{cen} A$ is noetherian and $A$ is a finitely generated $C$-module.

Definition 3.6. Let $(X, \mathscr{A})$ be noetherian.

(1) We denote by Ip $\mathscr{A}$ the full subcategory of $\mathscr{A}$-mod consisting of locally projective modules $\mathscr{P}$, i.e. such that $\mathscr{P}_{x}$ is a projective $\mathscr{A}_{x}$-module for every $x \in X$.

\footnotetext{
${ }^{3}$ Note that in this situation $\phi^{*}=\phi^{-1}$.
} 
(2) We say that $\mathscr{A}$ has enough locally projective modules if for every coherent $\mathscr{A}$-module $\mathscr{M}$ there is an epimorphism $\mathscr{P} \rightarrow \mathscr{M}$, where $\mathscr{P} \in \mathrm{lp} \mathscr{A}$. Since every quasi-coherent module is a sum of its coherent submodules, then for every quasi-coherent $\mathscr{A}$-module $\mathscr{M}$ there is an epmorphism $\mathscr{P} \rightarrow \mathscr{M}$, where $\mathscr{P}$ is a coproduct of modules from Ip $\mathscr{A}$.

An important example arises as follows. We say that a noetherian non-commutative scheme $(X, \mathscr{A})$ is quasi-projective if there is an ample $\mathscr{O}_{X}$-module $\mathscr{L}$ (Grothendieck, 1961, Section 4.5). Note that in this case $X$ is indeed a quasiprojective scheme over the ring $R=\bigoplus_{n=0}^{\infty} \Gamma\left(X, \mathscr{L}^{\otimes n}\right)$.

Proposition 3.7. Every quasi-projective non-commutative scheme $(X, \mathscr{A})$ has enough locally projective modules.

Proof. Let $\mathscr{L}$ be an ample $\mathscr{O}_{X}$-module, $\mathscr{M}$ be any coherent $\mathscr{A}$-module. There is an epimorphism of $\mathscr{O}_{X}$-modules $n \mathscr{O}_{X} \rightarrow \mathscr{M} \otimes_{\mathscr{O}_{X}} \mathscr{L}^{\otimes m}$ for some $m$, hence also an epimorphism $\mathscr{F}=n \mathscr{L}^{\otimes(-m)} \rightarrow \mathscr{M}$. Since $\operatorname{Hom}_{\mathscr{A}}\left(\mathscr{A} \otimes_{\mathscr{O}_{X}} \mathscr{F}, \mathscr{M}\right) \simeq \operatorname{Hom}_{\mathscr{O}_{X}}(\mathscr{F}, \mathscr{M})$, it gives an epimorphism of $\mathscr{A}$-modules $\mathscr{A} \otimes_{\mathscr{O}_{X}} \mathscr{F} \rightarrow \mathscr{M}$, where $\mathscr{A} \otimes_{\mathscr{O}_{X}} \mathscr{F} \in$ Ip $\mathscr{A}$.

We define an invertible $\mathscr{A}$-module as an $\mathscr{A}$-module $\mathscr{I}$ such that $\operatorname{End}_{\mathscr{A}} \mathscr{I} \simeq$ $\mathscr{A}^{\text {op }}$ and the natural map $\mathscr{H}_{0 m_{\mathscr{A}}}(\mathscr{I}, \mathscr{A}) \otimes \mathscr{A} \mathscr{I} \rightarrow\left(\operatorname{End}_{\mathscr{A}} \mathscr{I}\right)^{\text {op }} \simeq \mathscr{A}$ is an isomorphism. For instance, the modules constructed in the preceding proof are direct sums of invertible modules. On the contrary, one easily proves that, if $\mathscr{A}$ is noetherian and cen $\mathscr{A}=\mathscr{O}_{X}$, any invertible $\mathscr{A}$-module $\mathscr{I}$ is isomorphic to $\mathscr{A} \otimes_{\mathscr{O}_{X}} \mathscr{L}$, where $\mathscr{L}=\mathscr{H}_{\mathscr{A}} m_{\mathscr{A}-\mathscr{A}}(\mathscr{I}, \mathscr{I})$ and $\mathscr{L}$ is an invertible $\mathscr{O}_{X}$-module. (We will not use this fact.)

We denote by $\mathscr{C} \mathscr{A}$ the category of complexes of $\mathscr{A}$-modules, by $\mathscr{H} O \mathscr{A}$ the category of complexes modulo homotopy and by $\mathscr{D} \mathscr{A}$ the derived category $\mathscr{D}(\mathscr{A}$-Mod). We also use the conventional notations $\mathscr{C}^{\sigma} \mathscr{A} \mathscr{H}^{\sigma} \mathscr{A}$ and $\mathscr{D}^{\sigma} \mathscr{A}$, where $\sigma \in$ $\{+,-, b\}$. We denote by $\mathscr{D}^{\mathrm{c}} \mathscr{A}$ the full subcategory of compact objects $\mathscr{C} \bullet$ from $\mathscr{D} \mathscr{A}$, i.e. such that the natural morphism

$$
\bigsqcup_{i} \operatorname{Hom}_{\mathscr{D} \mathscr{A}}\left(\mathscr{C}_{\bullet}, \mathscr{F}_{i}\right) \rightarrow \operatorname{Hom}_{\mathscr{D} \mathscr{A}}\left(\mathscr{C}^{\bullet}, \bigsqcup_{i} \mathscr{F}_{i} \cdot\right)
$$

is bijective for any coproduct $\bigsqcup_{i} \mathscr{F}_{i}$.

Recall that a complex $\mathscr{I}^{\bullet}$ is said to be $K$-injective (Spaltenstein, 1988) if for every acyclic complex $\mathscr{C}^{\bullet}$ the complex $\operatorname{Hom}^{\bullet}\left(\mathscr{C}^{\bullet}, \mathscr{I}^{\bullet}\right)$ is acyclic too. We denote by $\mathrm{K}$-inj $\mathscr{A}$ the full subcategory of $\mathscr{H} o \mathscr{A}$ consisting of $\mathrm{K}$-injective complexes and by $\mathrm{K}$-inj ${ }_{0} \mathscr{A}$ its full subcategory consisting of acyclic K-injective complexes.

Proposition 3.8. Let $(X, \mathscr{A})$ be a non-commutative scheme (separated and quasicompact).

(1) For every complex $\mathscr{C} \bullet$ in $\mathscr{C} \mathscr{A}$ there is a $\mathrm{K}$-injective resolution, i.e. a $\mathrm{K}$ injective complex $\mathscr{I} \bullet \in \mathscr{C} \mathscr{A}$ together with a quasi-isomorphism $\mathscr{C} \bullet \rightarrow \mathscr{I} \bullet$.

(2) $\mathscr{D} \mathscr{A} \simeq \mathrm{K}-$ inj $\mathscr{A} / \mathrm{K}-\mathrm{inj}_{0} \mathscr{A}$.

Proof. As the category $\mathscr{A}$-Mod is a Grothendieck category, (1) follows immediately from (Alonso et al.,2000, Theorem 5.4) (see also (Spaltenstein, 1988, Lemma 
3.7 and Proposition 3.13)). Then (2) follows from (Spaltenstein, 1988, Proposition 1.5).

A complex $\mathscr{F} \bullet$ is said to be $K$-flat (Spaltenstein, 1988) if for every acyclic complex $\mathscr{S} \bullet$ of right $\mathscr{A}$-modules the complex $\mathscr{F} \bullet \otimes_{\mathscr{A}} \mathscr{S} \bullet$ is acyclic. The next result is quite analogous to (Alonso et al., 1997, Proposition 1.1) and the proof just repeats that of the cited paper with no changes.

Proposition 3.9. Let $(X, \mathscr{A})$ be a non-commutative scheme. Then for every complex $\mathscr{C} \bullet$ in $\mathscr{C} \mathscr{A}$ there is a $\mathrm{K}$-flat replica, i.e. a $\mathrm{K}$-flat complex $\mathscr{F} \bullet$ quasi-isomorphic to $\mathscr{C}^{\bullet}$.

Remark 3.10. If $(X, \mathscr{A})$ is noetherian and has enough locally projective modules, every complex from $\mathscr{C}^{-} \mathscr{A}$ has a locally projective (hence flat) resolution. Then (Spaltenstein, 1988, Theorem 3.4) implies that for every complex $\mathscr{C}$ from $\mathscr{C} \mathscr{A}$ there is an Lp-resolution, i.e. a K-flat complex $\mathscr{F} \bullet$ consisting of locally projective modules together with a quasi-isomorphism $\mathscr{F} \bullet \rightarrow \mathscr{C} \bullet$. For instance, it is the case if $(X, \mathscr{A})$ is quasi-projective (Proposition 3.7).

A complex $\mathscr{I}^{\bullet}$ is said to be weakly $\mathrm{K}$-injective if for every acyclic K-flat complex $\mathscr{F} \bullet$ the complex $\operatorname{Hom}^{\bullet}\left(\mathscr{F} \bullet, \mathscr{I}^{\bullet}\right)$ is exact.

Proposition 3.11. Spaltenstein, 1988, Propositions 5.4 and 5.15) Let $f:(X, \mathscr{A}) \rightarrow$ $(Y, \mathscr{B})$ be a morphism of non-commutative scheme.

(1) If $\mathscr{F} \bullet \in \mathscr{C} \mathscr{B}$ is $K$-flat, then so is also $f^{*} \mathscr{F} \bullet$ If, moreover, $\mathscr{F} \bullet$ is $K$-flat and acyclic, then $f^{*} \mathscr{F} \bullet$ is acyclic too.

(2) If $\mathscr{I} \in \mathscr{C} \mathscr{A}$ is weakly $K$-injective, then $f_{*} \mathscr{I}$ is weakly $K$-injective. If, moreover, $\mathscr{I}$ is weakly $K$-injective and acyclic, then $f_{*} \mathscr{I}$ is acyclic too.

Proposition 3.12. (Spaltenstein, 1988, Section 6) Let $(X, \mathscr{A})$ be a non-commutative scheme.

(1) The derived functors $\operatorname{RHom}_{\mathscr{A}}^{\bullet}(\mathscr{F} \bullet, \mathscr{G} \bullet)$ and $\mathrm{R} \mathscr{H}$ om $_{\mathscr{A}}^{\bullet}(\mathscr{F} \bullet, \mathscr{G} \bullet)$ exist and can be calculated using a $K$-injective resolution of $\mathscr{G} \bullet$ or a weakly $K$ injective resolution of $\mathscr{G} \bullet$ and a $K$-flat replica of $\mathscr{F} \bullet$.

(2) The derived functor $\mathscr{F} \bullet \stackrel{\mathrm{L}}{\otimes} \mathscr{\mathscr { A }} \cdot$, where $\mathscr{G} \bullet \in \mathscr{D}_{\mathscr{A}}^{\mathrm{op}}$, exists and can be calculated using a $K$-flat replica either of $\mathscr{F} \bullet$ or of $\mathscr{G} \bullet$. Moreover, if $\mathscr{G} \bullet$ is a complex of $\mathscr{A}-\mathscr{B}$-bimodules, where $\mathscr{B}$ is another sheaf of $\mathscr{O}_{X}$-algebras, there are isomorphisms of functors

$$
\begin{aligned}
& \operatorname{RHom}_{\mathscr{B}}\left(\mathscr{F} \cdot \stackrel{\mathrm{L}}{\otimes}_{\mathscr{A}} \mathscr{G} \bullet, \mathscr{M}^{\bullet}\right) \simeq \operatorname{RHom}_{\mathscr{A}}\left(\mathscr{F} \bullet, \operatorname{R} \mathscr{H}_{o m}\left(\mathscr{G} \cdot, \mathscr{M}^{\bullet}\right)\right) \\
& \mathrm{R} \mathscr{H}_{o m_{\mathscr{B}}}\left(\mathscr{F} \bullet \stackrel{\mathrm{L}}{\otimes}_{\mathscr{A}} \mathscr{G}^{\bullet}, \mathscr{M}^{\bullet}\right) \simeq \mathrm{R} \mathscr{H} o m_{\mathscr{A}}\left(\mathscr{F} \bullet, \mathrm{R} \mathscr{H}_{0 m_{\mathscr{B}}}\left(\mathscr{G}^{\bullet}, \mathscr{M}^{\bullet}\right)\right) \text {. }
\end{aligned}
$$

(3) For every morphism $f:(X, \mathscr{A}) \rightarrow(Y, \mathscr{B})$ the derived functors $\mathrm{L} f^{*}: \mathscr{D} \mathscr{B} \rightarrow$ $\mathscr{D} \mathscr{A}$ and $\mathrm{R} f_{*}: \mathscr{D} \mathscr{A} \rightarrow \mathscr{D} \mathscr{B}$ exist. They can be calculated using, respectively, $K$-flat replicas in $\mathscr{C} \mathscr{B}$ and weakly $K$-injective resolutions in $\mathscr{C} \mathscr{A}$. 
Moreover, there are isomorphisms of functors

$$
\begin{aligned}
& \operatorname{RHom}_{\mathscr{B}}^{\bullet}\left(\mathscr{F} \bullet, \mathrm{R} f_{*} \mathscr{G} \bullet\right) \simeq \operatorname{RHom}_{\mathscr{A}}^{\bullet}\left(\mathrm{L}^{*} \mathscr{F} \bullet, \mathscr{G} \bullet\right) \\
& \mathrm{R} \mathscr{H} o m_{\mathscr{B}}^{\bullet}\left(\mathscr{F} \bullet, \mathrm{R} f_{*} \mathscr{G} \bullet\right) \simeq \mathrm{R} f_{*} \mathrm{R} \mathscr{H} o m_{\mathscr{A}}^{\bullet}\left(\mathrm{L} f^{*} \mathscr{F} \bullet, \mathscr{G}^{\bullet}\right) \text {. }
\end{aligned}
$$

(4) If $g:(Y, \mathscr{B}) \rightarrow(Z, \mathscr{C})$ is another morphism of non-commutative schemes, then $\mathrm{L}(g f)^{*} \simeq \mathrm{L} f^{*}{ }^{\circ} \mathrm{L}^{*}$ and $\mathrm{R}(g f)_{*} \simeq \mathrm{R} g_{*}{ }^{\circ} \mathrm{R} f_{*}$.

If the considered non-commutative schemes have enough locally projective modules (for instance, are quasi-projective), one can replace in these statements $K$-flat replicas by Lp-resolutions.

In particular, let $f: A \rightarrow B$ be a homomorphism of rings. We consider $B$ as an algebra over a subring $S$ (an arbitrary one) of its center and $A$ as an algebra over a subring $R \subseteq \operatorname{cen} A \cap f^{-1}(S)$. Then we can identify $f$ with its sheafification $f^{\sim}$ : $\left(\operatorname{Spec} S, B^{\sim}\right) \rightarrow\left(\operatorname{Spec} R, A^{\sim}\right)$. In this context the functors $\left(f^{\sim}\right)^{*}$ and $\left(f^{\sim}\right)_{*}$ are just sheafifications, respectively, of the "back-up" functor ${ }_{B} M \mapsto{ }_{A} M$ and the "changeof-scalars" functor ${ }_{A} N \mapsto{ }_{B} B \otimes_{A} N$.

Definition 3.13. A complex $\mathscr{C} \bullet$ in $\mathscr{C} \mathscr{A}$ is said to be perfect if for every point $x \in X$ there is an open neighbourhood $U$ of $x$ such that $\left.\mathscr{C}\right|_{U}$ is quasi-isomorphic to a finite complex of locally projective coherent modules. We denote by Perf $\mathscr{A}$ the full subcategory of $\mathscr{D} \mathscr{A}$ consisting of perfect complexes.

The following result is well-known in commutative and affine cases (Neeman, 1996; Rouquier, 2008). Though the proof in non-commutative situation is almost the same, we include it for the sake of completeness. Actually, we reproduce the proof of Rouquier with slight changes.

Theorem 3.14. Let $(X, \mathscr{A})$ be a non-commutative scheme (quasi-compact and separated). Then $\mathscr{D} \mathscr{A}$ is compactly generated and $\mathscr{D}^{\mathrm{c}} \mathscr{A}=$ Perf $\mathscr{A}$.

Proof. Let $U \subseteq X$ be an open affine subset of $X, \mathscr{A}_{U}$ be the restriction of $\mathscr{A}$ onto $U, \complement U=X \backslash U, j=j_{U}: U \rightarrow X$ be the embedding. Then the inverse image functor $j^{*}: \mathscr{A}$-Mod $\rightarrow \mathscr{A}_{U}$-Mod is exact and the natural morphism $j^{*} j_{*} \rightarrow \mathbb{1}_{\mathscr{A}_{U} \text {-Mod }}$ is an isomorphism (actually, identity). Therefore Ker $j^{*}$ is a localizing subcategory and $\mathscr{A}_{U}$-Mod $\simeq \mathscr{A}$-Mod/Ker $j^{*}$. Note that $\operatorname{Ker} j_{U}^{*}$ consists of the $\mathscr{A}$-modules $\mathscr{M}$ such that $\operatorname{supp} \mathscr{M} \subseteq \complement U$. Then $\operatorname{KerL} j_{*}$ is a localizing subcategory of $\mathscr{D} \mathscr{A}$ and $\mathscr{D} \mathscr{A}_{U} \simeq \mathscr{D} \mathscr{A} / \mathrm{KerL} j_{*}$. This kernel coincide with the full subcategory $\mathscr{D} C \mathscr{A}$ of $\mathscr{D} \mathscr{A}$ consisting of complexes whose cohomologies are supported on $C U$.

If $W \subseteq X$ is another open affine subset, then the subcategories $\mathscr{D}_{C U} \mathscr{A}$ and $\mathscr{D}_{C W} \mathscr{A}$ intersect properly in the sense of (Rouquier, 2008, 5.2.3). Recall that it means that $j_{W}^{*} j_{U_{*}} j_{I J}^{*} \mathscr{F}=0$ as soon as $j_{W}^{*} \mathscr{F}=0$, what follows, for instance, from (Grothendieck, 1961, Corollaire (1.5.2)) applied to the cartesian diagram of affine 
morphisms (open embeddings)

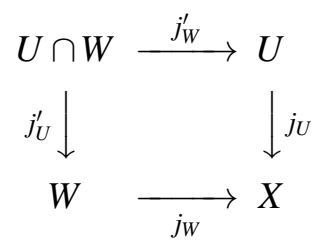

Therefore, if $X=\bigcup_{i=1}^{m} U_{i}$ is an open affine covering of $X$, then $\left\{\mathscr{D}_{\Gamma_{J_{i}}} \mathscr{A}\right\}$ is a cocovering of the triangulated category $\mathscr{D} \mathscr{A}$ as defined in (Rouquier, 2008, 5.3.3). If $S \subset\{1,2, \ldots, m\}$ does not contain $i, U_{S}=\bigcup_{j \in S} U_{j}$, then $\bigcap_{j \in S} \mathscr{D}_{C_{j}} \mathscr{A}=\mathscr{D}_{C_{S}} \mathscr{A}$ and the image of $\mathscr{D}_{C} U_{S} \mathscr{A}$ in $\mathscr{D} \mathscr{A}_{U_{i}}$ coincides with $\mathscr{D}_{U_{i} \backslash U_{S}} \mathscr{A}_{U_{i}}$. There are sections $f_{1}, f_{2}, \ldots, f_{k} \in A=\Gamma\left(U_{i}, \mathscr{O}_{X}\right)$ such that $U_{i} \backslash U_{S}=V\left(f_{1}, f_{2}, \ldots, f_{k}\right)$ as a closed subset of $U_{i}$. The following lemma shows that the subcategory $\mathscr{D}_{U_{i} \backslash U_{S}} \mathscr{A}_{U_{i}}$ is compactly generated in $\mathscr{D} \mathscr{A}_{U_{i}}$.

Lemma 3.15. Let $A$ be an algebra over a commutative ring $O$ and $I=\left(f_{1}, f_{2}, \ldots, f_{k}\right)$ be a finitely generated ideal in $O$. Let $K^{\bullet}(I)$ be the corresponding Koszul complex. Denote by $A-M_{I} d_{I}$ the full subcategory of A-Mod consisting of all modules $M$ such that for every element $a \in M$ there is $m$ such that $I^{m} a=0$. Denote by $\mathscr{D}_{I} A$ the full subcategory of $\mathscr{D A}$ consisting of all complexes such that their cohomologies belong to $A-\operatorname{Mod}_{I}$. Then $\mathscr{D}_{I} \mathscr{A}$ is generated by the complex $K_{A}^{\bullet}(I)=A \otimes_{O} K^{\bullet}(I)$.

Proof. Note that $\operatorname{Hom}_{\mathscr{D} A}\left(K_{\dot{A}}^{\bullet}(I), C^{\bullet}\right) \simeq \operatorname{Hom}_{\mathscr{D} O}\left(K^{\bullet}(I), C^{\bullet}\right)$ for every $C^{\bullet} \in \mathscr{D} A$. If $C^{\bullet} \in \mathscr{D}_{I} A$ is non-exact, then $\operatorname{Hom}_{\mathscr{D} O}\left(K^{\bullet}(I), C^{\bullet}[n]\right) \neq 0$ for some $n$ by (Rouquier, 2008, Proposition 6.6). It proves the claim.

Evidently, $K_{\dot{A}}(I)$ is compact in $\mathscr{D} A$. So we can now use (Rouquier, 2008, Theorem 5.15). It implies that $\mathscr{D} \mathscr{A}$ is compactly generated and a complex $\mathscr{C} \bullet$ in $\mathscr{D} \mathscr{A}$ is compact if and only if $j_{U_{i}}^{*} \mathscr{C} \bullet$ is compact in $\mathscr{D} \mathscr{A}_{U_{i}}$ for every $1 \leq i \leq m$. As $U_{i}$ is affine, compact complexes in $\mathscr{D} \mathscr{A}_{U_{i}}$ are just perfect complexes. Therefore, it is true for $\mathscr{D} \mathscr{A}$ too.

\section{MINORS}

Definition 4.1. Let $(X, \mathscr{B})$ be a non-commutative scheme, $\mathscr{P}$ be a locally projective and locally finitely generated $\mathscr{B}$-module, $\mathscr{A}=\left(\mathscr{E} n d_{\mathscr{B}} \mathscr{P}\right)^{\text {op }}$. The non-commutative scheme $(X, \mathscr{A})$ is called a minor of the non-commutative scheme $(X, \mathscr{B})]^{4}$

In this situation we consider $\mathscr{P}$ as $\mathscr{B}-\mathscr{A}$-bimodule (left over $\mathscr{B}$, right over $\mathscr{A}$ ). Let $\mathscr{P}^{\vee}=\mathscr{H}_{\text {om }_{\mathscr{B}}}(\mathscr{P}, \mathscr{B})$. It is an $\mathscr{A}$ - $\mathscr{B}$-bimodule, locally projective and locally finitely generated over $\mathscr{B}$. The following statements are evidently local, then they are well-known.

Proposition 4.2. The natural homomorphism $\mathscr{P} \rightarrow \mathscr{H}_{\text {om }} \mathscr{B}(\mathscr{P} \vee \mathscr{B})$ is an isomorphism. Moreover, $\mathscr{A} \simeq \mathscr{E} n d_{\mathscr{B}} \mathscr{P}^{\vee} \simeq \mathscr{P}^{\vee} \otimes_{\mathscr{B}} \mathscr{P}$.

\footnotetext{
${ }^{4}$ In the affine case this notion was introduced in (Drozd, 1973). Actually, the main results of this section are just global analogues of those from (Drozd, 1973).
} 
We consider the following functors:

$$
\begin{array}{r}
\mathrm{F}=\mathscr{P} \otimes_{\mathscr{A}}-: \mathscr{A} \text {-Mod } \rightarrow \mathscr{B} \text {-Mod, } \\
\mathrm{G}=\mathscr{H}_{\text {om }_{\mathscr{B}}}\left(\mathscr{P},{ }_{-}\right): \mathscr{B} \text {-Mod } \rightarrow \mathscr{A} \text {-Mod, } \\
\mathrm{H}=\mathscr{H} \text { om }_{\mathscr{A}}\left(\mathscr{P}^{\vee}{ }_{-}\right): \mathscr{A} \text {-Mod } \rightarrow \mathscr{B} \text {-Mod. }
\end{array}
$$

Note that $\mathrm{G}$ is exact and $\mathrm{G} \simeq \mathscr{P}^{\vee} \otimes_{\mathscr{B}-}$, so both $(\mathrm{F}, \mathrm{G})$ and $(\mathrm{G}, \mathrm{H})$ are adjoint pairs of functors. If the non-commutative scheme $(X, \mathscr{B})$ is noetherian, so is also $(X, \mathscr{A})$ and these functors map coherent sheaves to coherent ones.

We set $\mathscr{I}_{\mathscr{P}}=\operatorname{Im}\left\{\mu_{\mathscr{P}}: \mathscr{P} \otimes_{\mathscr{A}} \mathscr{P}^{\vee} \rightarrow \mathscr{B}\right\}$, where $\mu(p \otimes \gamma)=\gamma(p)$.

Theorem 4.3. (1) $\mathrm{G}$ is a bilocalization functor, thus $\mathscr{C}$ is a bilocalizing subcategory, $\mathscr{A}-\mathrm{Mod} \simeq \mathscr{B}-\mathrm{Mod} / \mathscr{C}$, where $\mathscr{C}=\mathrm{KerF}=\mathscr{P} \perp$ and both $\mathrm{F}$ and $\mathrm{H}$ are full embeddings $\mathscr{A}$-Mod $\rightarrow \mathscr{B}$-Mod (usually with different images).

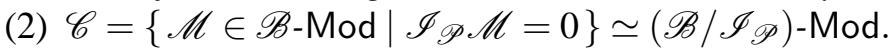

(3) $\mathrm{Im} \mathrm{F}=\perp_{\mathscr{C}}$ coincides with the full subcategory of $\mathscr{B}-\mathrm{Mod}$ consisting of all modules $\mathscr{M}$ such that for every point $x \in X$ there is an exact sequence $P_{1} \rightarrow P_{0} \rightarrow \mathscr{M}_{x} \rightarrow 0$, where $P_{0}, P_{1}$ are multiples of $\mathscr{P}_{x}$ (i.e. direct sums, maybe infinite, of its copies). We denote this subcategory by $\mathscr{P}$-Mod.

$3^{\prime} . \operatorname{ImH}=\mathscr{C}^{\perp}$ coinsides with the full subcategory of $\mathscr{B}$-Mod consisting of all modules $\mathscr{M}$ such that there is an exact sequence $0 \rightarrow \mathscr{M} \rightarrow \mathscr{I}_{0} \rightarrow \mathscr{I}_{1}$, where $\mathscr{I}_{i} \in \mathrm{H}(\mathscr{A}$-Inj $)\left[5\right.$ We denote this subcategory by $\mathscr{P}^{\ln }{ }_{\text {_Mod. }}$

Proof. Theorem 2.1 and Corollary 2.3 show that, to prove claims 1,3 and $3^{\prime}$, it is enough to prove the following statements.

Proposition 4.4. (1) The natural morphism $\phi: \mathbb{1}_{\mathscr{A}-\operatorname{Mod}} \rightarrow \mathrm{G} \circ \mathrm{F}$ is an isomorphism.

(2) $\operatorname{ImF}=\mathscr{P}$-Mod.

$2^{\prime} . \operatorname{Im} \mathrm{H}=\mathscr{P}^{\text {Inj }}{ }_{-M o d}$.

Proof. As the claims 1 and 2 are local, we can suppose that the non-commutative scheme $(X, \mathscr{B})$ is affine, so replace $\mathscr{B}$-Mod by $B$-Mod, where $B=\Gamma(X, \mathscr{B})$. Then $\mathscr{P}=P^{\sim}$ for some finitely generated projective $B$-module and $\mathscr{A}=A^{\sim}$, where $A=\left(\operatorname{End}_{B} P\right)^{\text {op }}$. Hence we can also replace $\mathscr{A}$-Mod by $A$-Mod and $\mathscr{P}$-Mod by $P$-Mod, the full subcategory of $B$-Mod consisting of all modules $N$ such that there is an exact sequence $P_{1} \rightarrow P_{0} \rightarrow N \rightarrow 0$, where $P_{i}$ are multiples of $P$.

Obviously, $\phi(A)$ is an isomorphism. Since $\mathrm{F}$ and $\mathrm{G}$ preserve arbitrary coproducts, $\phi(F)$ is an isomorphism for any free $A$-module $F$. Let $M \in \mathscr{A}$-Mod. There is an exact sequence $F_{1} \rightarrow F_{0} \rightarrow M \rightarrow 0$, where $F_{0}, F_{1}$ are free modules, which gives rise to a commutative diagram with exact rows

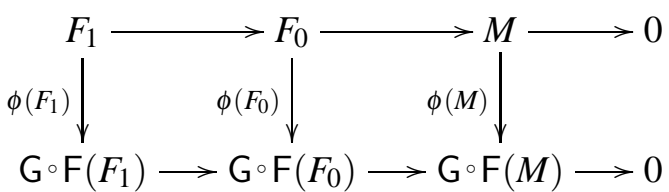

\footnotetext{
${ }^{5}$ Note that all $\mathscr{B}$-modules from $\mathrm{H}(\mathscr{A}$-Inj) are injective.
} 
As the first two vertical arrows are isomorphisms, so is $\phi(M)$, which proves claim 1. Moreover, we get an exact sequence $\mathrm{F}\left(F_{1}\right) \rightarrow \mathrm{F}\left(F_{0}\right) \rightarrow \mathrm{F}(M) \rightarrow 0$, where $\mathrm{F}\left(F_{i}\right)$ are multiples of $\mathrm{F}(A)=P$. Therefore, $\mathrm{F}(M) \in P$-Mod.

Consider now the natural morphism $\psi: \mathrm{F} \circ \mathrm{G} \rightarrow \mathbb{1}_{B \text {-Mod. }}$. This time $\psi(P)$ is an isomorphism. Let now $N$ be a $B$-module such that there is an exact sequence $P_{1} \rightarrow P_{0} \rightarrow N \rightarrow 0$, where $P_{i}$ are multiples of $P$. Then there is a commutative diagram with exact rows

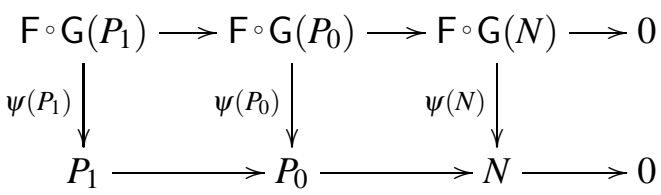

The first two vertical arrows are isomorphisms, so $\psi(N)$ is also an isomorphism. It proves claim 3.

The proof of $3^{\prime}$ is quite analogous to that of 3 , so we omit it.

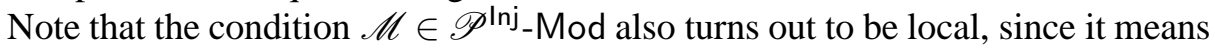
that the natural map $\mathscr{M} \rightarrow \mathrm{H} \circ \mathrm{G}(\mathscr{M})$ is an isomorphism.

The statement 2 is also local, so we only have to prove it for a ring $B$, a finitely generated projective $B$-module $P$ and the ideal $I_{P}=\operatorname{Im} \mu_{P}$. It follows from (Cartan and Eilenberg, 1956, Proposition VII.3.1) that $I_{P} P=P$. Therefore, if $f: P \rightarrow M$ is non-zero, then $I_{P} \operatorname{Im} f=\operatorname{Im} f \neq 0$, hence $I_{P} M \neq 0$. On the contrary, if $I_{P} M \neq 0$, there is an element $u \in M$, elements $p_{i} \in P$ and homomorphisms $\gamma_{i}: P \rightarrow B$ such that $\sum_{i} \gamma_{i}\left(p_{i}\right) u \neq 0$. Let $\beta: B \rightarrow M$ maps 1 to $u$ and $\gamma_{i}^{u}=\beta \gamma_{i}$. Then at least one of the homomorpisms $\gamma_{i}^{u}$ is non-zero.

The functor $\mathrm{G}$ is exact, so it induces a functor $\mathrm{DG}: \mathscr{D} \mathscr{B} \rightarrow \mathscr{D} \mathscr{A}$ mapping a complex $\mathscr{F} \bullet$ to $G \mathscr{F} \bullet$. It is both left and right derived functor of $\mathrm{G}$. We can also consider the left derived functor LF of $\mathrm{F}$ and the right derived functor $\mathrm{RH}$ of $\mathrm{H}$, both being functors $\mathscr{D} \mathscr{A} \rightarrow \mathscr{D} \mathscr{B}$. Obviously, DG maps $\mathscr{D}^{\sigma} \mathscr{B}$ to $\mathscr{D}^{\sigma} \mathscr{A}$, where $\sigma \in\{+,-, b\}$, LF maps $\mathscr{D}^{-} \mathscr{A}$ to $\mathscr{D}^{-} \mathscr{B}$ and RH maps $\mathscr{D}^{+} \mathscr{A}$ to $\mathscr{D}^{+} \mathscr{B}$.

Theorem 4.5. (1) The functors (LF, DG) and (DG, RH) form adjoint pairs.

(2) DG is a bilocalization functor, KerDG $=\mathscr{D}_{\mathscr{C}} \mathscr{B}$, where $\mathscr{C}=\operatorname{KerG}$ is a bilocalizing subcategory, $\mathscr{D} \mathscr{A} \simeq \mathscr{D} \mathscr{B} / \mathscr{D}_{\mathscr{C}} \mathscr{B}$ and both $\mathrm{LF}$ and $\mathrm{RH}$ are full embeddings $\mathscr{D} \mathscr{A} \rightarrow \mathscr{D} \mathscr{B}$ (usually with different images).

(3) The functor LF maps $\mathscr{D}^{\mathrm{c}} \mathscr{A}$ to $\mathscr{D}^{\mathrm{c}} \mathscr{B}$.

(4) (KerDG,ImLF) as well as (Im RH, KerDG) are semi-orthogonal decompositions of $\mathscr{D} \mathscr{B}$.

(5) $\operatorname{ImLF}={ }^{\perp}\left(\mathscr{D}_{\mathscr{C}} \mathscr{B}\right)$ coincides with the full subcategory $\mathscr{D} \mathscr{P}$ of $\mathscr{D} \mathscr{B}$ consisting of complexes quasi-isomorphic to $K$-flat complexes $\mathscr{F} \bullet$ such that for every $x \in X$ and every component $\mathscr{F}^{i}$ the localization $\mathscr{F}_{x}^{i}$ is a direct limit of modules from add $\mathscr{P}_{x}$. The same is true if we replace $\mathscr{D}$ by $\mathscr{D}^{-}$.

5p. If $\mathscr{A}$ and $\mathscr{B}$ have enough locally projective modules (for instance, if $X$ is quasi-projective), ImLF coincides with the full subcategory $\mathscr{D} \mathscr{P}$ of $\mathscr{D} \mathscr{B}$ consisting of complexes quasi-isomorphic to $K$-flat complexes $\mathscr{F} \bullet$ such that 
$\mathscr{F}_{x}^{i} \in \operatorname{Add} \mathscr{P}_{x}$ for every $i \in \mathbb{Z}$ and every point $x \in X$. The same is true if we replace $\mathscr{D}$ by $\mathscr{D}^{-}$.

5'. Im RH $=\left(\mathscr{D}_{\mathscr{C}} \mathscr{B}\right)^{\perp}$ coincides with the full subcategory $\mathscr{D} \mathscr{P}^{\mathrm{lnj}}$ of $\mathscr{D} \mathscr{B}$ consisting of complexes quasi-isomorphic to $K$-injective complexes consisting of modules from $\mathrm{H}(\mathscr{A}$-Inj $)$. The same is true if we replace $\mathscr{D}$ by $\mathscr{D}^{+}$.

Note that the condition in $5^{\prime}$ can also be verified locally at every point $x \in X$.

Proof. 1. Let $\mathscr{F} \bullet$ be a K-flat replica of $\mathscr{M}^{\bullet} \in \mathscr{D} \mathscr{A}$ and $\mathscr{I} \bullet$ be an injective resolution of $\mathscr{N}^{\bullet} \in \mathscr{D} \mathscr{B}$. Then $\mathrm{LF} \mathscr{M}^{\bullet}=\mathrm{F} \mathscr{F} \bullet$ and DG $\mathscr{N}^{\bullet}=\mathrm{G} \mathscr{I} \bullet$. As $\mathscr{P} \in \mathrm{lp} \mathscr{B}$, the complex $\mathrm{F} \mathscr{F} \bullet$ is $\mathrm{K}$-flat and the complex $\mathrm{G} \mathscr{I} \bullet$ is K-injective. By Proposition 3.122,

$$
\begin{aligned}
\operatorname{RHom}_{\mathscr{B}}\left(\mathrm{F} \mathscr{F}^{\bullet}, \mathscr{I}^{\bullet}\right)= & \operatorname{Hom}_{\mathscr{B}}^{\bullet}\left(\mathrm{F} \mathscr{F} \cdot, \mathscr{I}^{\bullet}\right) \simeq \\
& \operatorname{Hom}_{\mathscr{A}}\left(\mathscr{F} \bullet, G \mathscr{I}^{\bullet}\right)=\operatorname{RHom}_{\mathscr{A}}\left(\mathscr{F} \bullet, G \mathscr{I}^{\bullet}\right) .
\end{aligned}
$$

Taking zero cohomologies, we obtain that

$$
\operatorname{Hom}_{\mathscr{B}}\left(\mathrm{F} \mathscr{F}^{\bullet}, \mathscr{I}^{\bullet}\right) \simeq \operatorname{Hom}_{\mathscr{A}}\left(\mathscr{F}^{\bullet}, \mathrm{G} \mathscr{I}^{\bullet}\right) .
$$

Choose now a K-flat replica $\mathscr{G}^{\bullet}$ of $\mathscr{N}^{\bullet}$ and a K-injective resolution $\mathscr{J}^{\bullet}$ of $\mathscr{M}^{\bullet}$. Then DG $\mathscr{N}^{\bullet}=\mathrm{G} \mathscr{G} \bullet$ and $\mathrm{RH} \mathscr{M}^{\bullet}=\mathrm{H} \mathscr{J} \bullet$. By (Spaltenstein, 1988, Proposition 5.14), $\mathrm{H} \mathscr{J} \bullet$ is weakly K-injective. By Proposition 3.122 and (Spaltenstein, 1988, Proposition 6.1),

$$
\begin{aligned}
\operatorname{RHom}_{\mathscr{A}}\left(\mathrm{G} \mathscr{G} \bullet, \mathscr{J}^{\bullet}\right)=\operatorname{Hom}_{\mathscr{A}}^{\bullet}\left(\mathrm{G}^{\bullet} \cdot, \mathscr{J}^{\bullet}\right) \simeq \\
\qquad \operatorname{Hom}_{\mathscr{B}}(\mathscr{G} \cdot \mathrm{H} \mathscr{J} \bullet)=\operatorname{RHom}_{\mathscr{B}}(\mathscr{G} \cdot \mathrm{H} \mathscr{J} \bullet) .
\end{aligned}
$$

Taking zero cohomologies, we obtain that

$$
\operatorname{Hom}_{\mathscr{A}}\left(\mathrm{G} \mathscr{G} \bullet, \mathscr{J}^{\bullet}\right) \simeq \operatorname{Hom}_{\mathscr{B}}\left(\mathscr{G}^{\bullet}, \mathrm{H} \mathscr{J}^{\bullet}\right)
$$

2 follows now from Theorem 4.3 and Theorem 2.1 The statement 4 follows from Theorem 2.6 .

3. As the right adjoint DG of LF preserves arbitrary coproducts, LF maps compact objects to compact ones.

4 follows now from Corollary 2.6 .

5. The construction of (Alonso et al., 1997, Proposition 1.1) gives for any complex $\mathscr{M}^{\bullet} \in \mathscr{D} \mathscr{A}$ a quasi-isomorphic K-flat complex $\mathscr{F}^{\bullet}$ such that all its components $\mathscr{F}^{i}$ are flat. Moreover, $\mathscr{F}^{\bullet}$ is left bounded if so is $\mathscr{M}^{\bullet}$. By (Bourbaki, 1980, Ch.X, $\S 1$, Théorème 1), $\mathscr{F}_{x}^{i} \simeq \lim \mathscr{L}_{n}^{i}$, where $\mathscr{L}_{n}^{i}$ are projective finitely generated $\mathscr{A}_{x}$-modules, hence belong to add $\mathscr{A}_{x}$. Then LF $\mathscr{M}^{\bullet} \simeq \mathrm{F} \mathscr{F} \bullet$. As F preserves direct limits and $\mathrm{F} \mathscr{A} \simeq \mathscr{P}, \mathrm{F} \mathscr{F}_{x}^{i} \simeq \underline{\lim } \mathrm{F} \mathscr{L}_{n}^{i}$ and $\mathrm{F} \mathscr{L}_{n}^{i} \in$ add $\mathscr{P}_{x}$. Hence $\mathscr{M}^{\bullet} \in \mathscr{D} \mathscr{P}$.

On the contrary, let $\mathscr{N} \bullet \in \mathscr{D} \mathscr{P}$. We can suppose that it is K-flat and for every $i \in \mathbb{Z}$ and every $x \in X$ we can present $\mathscr{N}_{x}^{i}$ as $\lim \mathscr{N}_{n}^{i}$, where $\mathscr{N}_{n}^{i} \in \operatorname{add} \mathscr{P}_{x}$. Then the complex G $\mathscr{N}^{\bullet}$ is also K-flat (Spaltenstein, 1988, Proposition 5.4), so $\mathrm{LF} \circ \operatorname{DG}\left(\mathscr{N}^{\bullet}\right) \simeq \mathrm{FG}\left(\mathscr{N}^{\bullet}\right)$. As the natural map $\mathrm{FG}(\mathscr{P}) \rightarrow \mathscr{P}$ is an isomorphism, the same is true for all modules $\mathscr{N}_{n}^{i}$, hence also for $\mathscr{N}_{x}^{i}$. Therefore, the natural map $\operatorname{LF} \circ \mathrm{DG}(\mathscr{N}) \rightarrow \mathscr{N}$ is an isomorphism. 
The proof of $5 p$ is quite analogous to the proof of 5 , taking into account that in this situation every complex is quasi-isomorphic to a K-flat complex of locally projective modules. The proof of $5^{\prime}$ is also analogous to that of 5 .

Recall that $\mathscr{C}=\operatorname{Ker} G \simeq\left(\mathscr{B} / \mathscr{I}_{\mathscr{P}}\right)$-Mod. There is one special case when the category KerDG can be described analogously.

Theorem 4.6. Suppose that the ideal $\mathscr{I}_{\mathscr{P}}$ is flat as a right $\mathscr{B}$-module. Then $\operatorname{KerDG} \simeq \mathscr{D}\left(\mathscr{B} / \mathscr{I}_{\mathscr{P}}\right)$.

Proof. Let $\mathscr{I}=\mathscr{I}_{\mathscr{P}}, \mathscr{Q}=\mathscr{B} / \mathscr{I}$. One easily sees that $\mathscr{I}^{2}=\mathscr{I}$. We identify $\mathscr{D} \mathscr{Q}$ with the full triangulated subcategory of $\mathscr{D} \mathscr{B}$, obviously contained in KerDG. Let $\mathscr{F} \bullet \in$ KerDG, i.e. its cohomologies are indeed $\mathscr{Q}$-modules. We can suppose that $\mathscr{F} \bullet$ is K-flat. Tensoring it with the exact sequence $0 \rightarrow \mathscr{I} \rightarrow \mathscr{B} \rightarrow \mathscr{Q} \rightarrow 0$, we obtain an exact sequence of complexes $0 \rightarrow \mathscr{I} \otimes_{\mathscr{B}} \mathscr{F} \bullet \rightarrow \mathscr{F} \bullet \rightarrow \mathscr{Q} \otimes_{\mathscr{B}} \mathscr{F} \bullet \rightarrow 0$. Since $\mathscr{I}$ is flat, $H^{\bullet}\left(\mathscr{I} \otimes_{\mathscr{B}} \mathscr{F}^{\bullet}\right) \simeq \mathscr{I} \otimes_{\mathscr{B}} H^{\bullet}(\mathscr{F} \bullet)$. Note that $\mathscr{I} \otimes_{\mathscr{B}} \mathscr{Q} \simeq \mathscr{I} / \mathscr{I}^{2}=$ 0 , whence $\mathscr{I} \otimes_{\mathscr{B}} \mathscr{M}=0$ for any $\mathscr{Q}$-module. Therefore, $H^{\bullet}\left(\mathscr{I} \otimes_{\mathscr{B}} \mathscr{F} \bullet\right)=0$, hence $\mathscr{F}^{\bullet}$ is quasi-isomorphic to $\mathscr{Q} \otimes_{\mathscr{B}} \mathscr{F}^{\bullet}$, which is in $\mathscr{D} \mathscr{Q}$.

Example 4.7. An important special case of minors appears as the endomorphism construction. Let $\mathscr{A}$ be a non-commutative scheme, $\mathscr{F}$ be a coherent $\mathscr{A}$-module and $\mathscr{A}_{\mathscr{F}}=\mathscr{E} n d_{\mathscr{A}}(\mathscr{A} \oplus \mathscr{F})^{\text {op }}$. Then $\mathscr{A}_{\mathscr{F}}$ is identified with the algebra of matrices

$$
\mathscr{A}_{\mathscr{F}}=\left(\begin{array}{ll}
\mathscr{A} & \mathscr{F} \\
\mathscr{F}^{\prime} & \mathscr{E}
\end{array}\right)
$$

where $\mathscr{F}^{\prime}=\mathscr{H}_{o m_{\mathscr{A}}}(\mathscr{F}, \mathscr{A})$ and $\mathscr{E}=\left(\mathscr{E} n d_{\mathscr{A}} \mathscr{F}\right)^{\text {op }}$. If $\mathscr{P}_{\mathscr{F}}=\left(\frac{\mathscr{A}}{\mathscr{F}^{\prime}}\right)$ considered as $\mathscr{A}_{\mathscr{F}}$-module, then $\mathscr{A} \simeq\left(\mathscr{E}_{n} d_{\mathscr{A} \mathscr{F}} \mathscr{P}_{\mathscr{F}}\right)^{\text {op }}$, so $\mathscr{A}$ is a minor of $\mathscr{A}_{\mathscr{F}}$ and the categories $\mathscr{A}$-Mod and $\mathscr{D} \mathscr{A}$ are bilocalizations, respectively, of $\mathscr{A}_{\mathscr{F}}$-Mod and $\mathscr{D} \mathscr{A}_{\mathscr{F}}$. The corresponding functors are

$$
\begin{aligned}
\mathrm{F}_{\mathscr{F}} & =\mathscr{P}_{\mathscr{F}} \otimes_{\mathscr{A}}, \\
\mathrm{G}_{\mathscr{F}} & =\mathscr{H}_{o m_{\mathscr{F}}}\left(\mathscr{P}_{\mathscr{F}},_{-}\right), \\
\mathrm{H}_{\mathscr{F}} & =\mathscr{H}_{o m_{\mathscr{F}}}\left(\mathscr{P}_{\mathscr{F}}{ }_{-}\right) .
\end{aligned}
$$

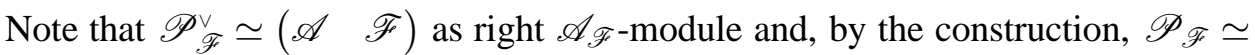
$\mathscr{H}_{\mathscr{A}}\left(\mathscr{P}_{\mathscr{F}}, \mathscr{A}\right)$. Theorem 4.3 2 then implies that the kernel $\mathscr{C}$ of the functor $\mathrm{G}_{\mathscr{F}}: \mathscr{A}_{\mathscr{F}}$-Mod $\rightarrow \mathscr{A}$-Mod is equivalent to $\mathscr{E} / \mathscr{I}_{\mathscr{F}}$-Mod, where $\mathscr{I}_{\mathscr{F}}$ is the image of the natural map $\mathscr{F}^{\prime} \otimes_{\mathscr{A}} \mathscr{F} \rightarrow \mathscr{E}$. This construction will be crucial in Section 8

\section{HEREDITY CHAINS}

We consider an application of minors to global dimensions and semi-orthogonal decompositions. Let $(X, \mathscr{B})$ be a non-commutative scheme, $\mathscr{M}$ be a $\mathscr{B}$-module. We call sup $\left\{i \mid \mathscr{E} x t_{\mathscr{B}}^{i}\left(\mathscr{M},{ }_{-}\right) \neq 0\right\}$ the local projective dimension of the $\mathscr{B}$-module $\mathscr{M}$ and denote it by $\operatorname{lp} \cdot \operatorname{dim}_{\mathscr{B}} \mathscr{M}$. If $(X, \mathscr{B})$ is noetherian and $\mathscr{M}$ is coherent, then $\operatorname{lp} \cdot \operatorname{dim}_{\mathscr{B}} \mathscr{M}=\sup \left\{\operatorname{pr} \cdot \operatorname{dim}_{\mathscr{B}_{x}} \mathscr{M}_{x} \mid x \in X\right\}$. 
Lemma 5.1. Let $(X, \mathscr{B})$ be a non-commutative scheme, $\mathscr{P}$ be a locally projective and locally finitely generated $\mathscr{B}$-module, $\mathscr{A}=(\mathscr{E} \text { nd } \mathscr{B} \mathscr{P})^{\mathrm{op}}$ and $\overline{\mathscr{B}}=\mathscr{B} / \mathscr{I}_{\mathscr{P}}$. Suppose that $\mathscr{P}$ is flat as right $\mathscr{A}$-module,

$$
\begin{aligned}
\operatorname{lp} \cdot \operatorname{dim}_{\mathscr{B}} \mathscr{I}_{\mathscr{P}} & =d, \\
\operatorname{gl} \operatorname{dim} \mathscr{A} & =n, \\
\operatorname{gl} \operatorname{dim} \overline{\mathscr{B}} & =m .
\end{aligned}
$$

Then gl.dim $\mathscr{B} \leq \max \{m+d+2, n\}$.

Proof. Let $\overline{\mathscr{B}}=\mathscr{B} / \mathscr{I}_{\mathscr{P}}$. Then lp. $\operatorname{dim}_{\mathscr{B}} \overline{\mathscr{B}}=d+1$, and from the spectral sequence $\operatorname{Ext}_{\mathscr{\mathscr { B }}}^{p}\left(\mathscr{M}, \mathscr{E} x t_{\mathscr{B}}^{q}\left(\overline{\mathscr{B}},_{-}\right)\right) \Rightarrow \operatorname{Ext}_{\mathscr{B}}^{p+q}\left(\mathscr{M},_{-}\right)$it follows that pr.dim $\operatorname{dim}_{\mathscr{B}} \mathscr{M} \leq m+d+1$ for every $\overline{\mathscr{B}}$-module $\mathscr{M}$. Consider the functors $\mathrm{G}=\operatorname{Hom}_{\mathscr{B}}\left(\mathscr{P},{ }_{-}\right)$and $\mathrm{F}=\mathscr{P} \otimes_{\mathscr{A}-}$. Since the morphism GFG $\rightarrow$ G, arising from the adjunction, is an isomorphism, the kernel and the cokernel of the natural map $\alpha:$ FG $\mathscr{M} \rightarrow \mathscr{M}$ are annihilated by G, so are actually $\overline{\mathscr{B}}$-modules. It implies that $\operatorname{Ext}_{\mathscr{B}}^{i}(\mathscr{M}, \mathscr{N}) \simeq \operatorname{Ext}_{\mathscr{B}}^{i}(\mathrm{FG} \mathscr{M}, \mathscr{N})$ if $i>$ $m+d+2$, so pr.dim $\operatorname{dim}_{\mathscr{B}} \mathscr{M} \leq \max \left\{m+d+2\right.$, pr.dim $\left.\operatorname{din}_{\mathscr{B}} \mathrm{FG} \mathscr{M}\right\}$. As both functors $\mathrm{F}$ and $\mathrm{G}$ are exact, $\operatorname{Ext}_{\mathscr{B}}^{i}\left(\mathrm{~F}_{-},{ }_{-}\right) \simeq \operatorname{Ext}_{\mathscr{A}}^{i}\left({ }_{-}, \mathrm{G}_{-}\right)$, so pr.dim $\mathscr{B}_{\mathscr{B}} \mathrm{FG} \mathscr{M} \leq n$.

Definition 5.2. (1) Let $(X, \mathscr{B})$ and $(X, \mathscr{A})$ be two non-commutative schemes. A relating chain between $\mathscr{B}$ and $\mathscr{A}$ is a sequence $\left(\mathscr{B}_{1}, \mathscr{P}_{1}, \mathscr{B}_{2}, \mathscr{P}_{2}, \ldots, \mathscr{P}_{r}, \mathscr{B}_{r+1}\right)$, where $\mathscr{B}_{1}=\mathscr{B}, \mathscr{B}_{r+1}=\mathscr{A}$, every $\mathscr{P}_{i}(1 \leq i \leq r)$ is a locally projective and locally finitely generated $\mathscr{B}_{i}$-module which is also flat as right $\mathscr{A}_{i}$-module, where $\mathscr{A}_{i}=\left(\mathscr{E} n d_{\mathscr{B}_{i}} \mathscr{P}_{i}\right)^{\text {op }}$, and $\mathscr{B}_{i+1}=\mathscr{B}_{i} / \mathscr{I}_{\mathscr{P}_{i}}$ for $1 \leq i \leq r$.

(2) The relating chain is said to be flat if, for every $1 \leq i \leq r, \mathscr{I}_{\mathscr{P}_{i}}$ is flat as right $\mathscr{B}_{i}$-module. Note that it is the case if the natural map $\mathscr{P}_{i} \otimes_{\mathscr{A}_{i}} \mathscr{P}_{i}^{\vee} \rightarrow \mathscr{B}_{i}$ is a monomorphism.

(3) The relating chain is said to be pre-heredity if, for every $1 \leq i \leq r, \mathscr{I}_{\mathscr{P}_{i}}$ is locally projective as left $\mathscr{B}_{i}$-module. If it is both pre-heredity and flat, it is said to be heredity.

(4) If the relating chain is heredity and all non-commutative schemes $\mathscr{A}_{i}$ are hereditary, i.e. gl.dim $\mathscr{A}_{i} \leq 1$, we say that the non-commutative scheme $\mathscr{B}$ is quasi-hereditary of level $r$. (Thus quasi-hereditary of level 0 means hereditary).

We fix a relating chain $\left(\mathscr{B}_{1}, \mathscr{P}_{1}, \mathscr{B}_{2}, \mathscr{P}_{2}, \ldots, \mathscr{P}_{r}, \mathscr{B}_{r+1}\right)$ between $\mathscr{B}$ and $\mathscr{A}$ and keep the notations of Definition 5.21 .

Corollary 5.3. Let gl.dim $\mathscr{A}_{i} \leq n$ and $\operatorname{lp} \cdot \operatorname{dim}_{\mathscr{B}_{i}} \mathscr{I}_{\mathscr{P}_{i}} \leq d$ for all $1 \leq i \leq r$. Then gl.dim $\mathscr{B} \leq r(d+2)+\max \{\mathrm{gl} \cdot \operatorname{dim} \mathscr{A}, n-d-2\}$. If this relating chain is preheredity, then gl.dim $\mathscr{B} \leq \operatorname{gl} . \operatorname{dim} \mathscr{A}+2 r$.

Using Theorem 4.5(4), Theorem 4.6 and induction, we also get the following result.

Corollary 5.4. If this relating chain is flat, there are semi-orthogonal decompositions $\left(\mathscr{T}, \mathscr{T}_{r}, \ldots, \mathscr{T}_{1}\right)$ and $\left(\mathscr{T}_{1}^{\prime}, \mathscr{T}_{2}^{\prime}, \ldots, \mathscr{T}_{r}^{\prime}, \mathscr{T}\right)$ of $\mathscr{D} \mathscr{B}$ such that $\mathscr{T}_{i} \simeq \mathscr{T}_{i}^{\prime} \simeq \mathscr{D} \mathscr{A}_{i}$ $(1 \leq i \leq r)$ and $\mathscr{T} \simeq \mathscr{D} \mathscr{A}$. 
Note that, as a rule, $\mathscr{T}_{i} \neq \mathscr{T}_{i}^{\prime}$.

Corollary 5.5. If a non-commutative scheme $\mathscr{B}$ is quasi-hereditary of level $r$, then gl.dim $\mathscr{B} \leq 2 r+1$ and there are semi-orthogonal decompositions $\left(\mathscr{T}, \mathscr{T}_{r}, \ldots, \mathscr{T}_{1}\right)$ and $\left(\mathscr{T}_{1}^{\prime}, \mathscr{T}_{2}^{\prime}, \ldots, \mathscr{T}_{r}^{\prime}, \mathscr{T}\right)$ of $\mathscr{D} \mathscr{B}$ such that $\mathscr{T}_{i} \simeq \mathscr{T}_{i}^{\prime}(1 \leq i \leq r)$, as well as $\mathscr{T}$, is equivalent to the derived category of a hereditary non-commutative scheme.

Remark 5.6. Suppose that $(X, \mathscr{B})$ is affine: $X=\operatorname{Spec} R$ and $\mathscr{B}=B^{\sim}$.

(1) If $B$ is semiprimary, then $\mathscr{B}$ is quasi-hereditary with respect to our definition if and only if $B$ is quasi-hereditary in the classical sense of (Cline et al., 1988; Dlab and Ringel, 1989).

(2) If $R$ is a discrete valuation ring and $B$ is an $R$-order in a separable algebra, then $\mathscr{B}$ is quasi-hereditary with respect to our definition if and only if $B$ is quasi-hereditary in the sense of (König, 1990).

Example 5.7. Consider the endomorphism construction of Example 4.7 Suppose that $\mathscr{F}$ is flat as right $\mathscr{E}$-module, $\mathscr{F}^{\prime}$ is locally projective as left $\mathscr{E}$-module and the natural map $\mu_{\mathscr{F}}: \mathscr{F} \otimes_{\mathscr{E}} \mathscr{F}^{\prime} \rightarrow \mathscr{A}$ is a monomorphism. Let $\tilde{\mathscr{P}}=\left(\begin{array}{c}\mathscr{F} \\ \mathscr{E}\end{array}\right)$ and $\overline{\mathscr{A}}=\mathscr{A} / \operatorname{Im} \mu_{\mathscr{F}}$. Then one can easily verify that $(\mathscr{A} \mathscr{F}, \tilde{\mathscr{P}}, \overline{\mathscr{A}})$ is a heredity relating chain. Therefore, if both $\mathscr{E}$ and $\overline{\mathscr{A}}$ are quasi-hereditary, so is $\mathscr{A}_{\mathscr{F}}$. These conditions hold, for instance, if $\mathscr{A}$ is noetherian and reduced, $\mathscr{F}$ is coherent torsion free and $\mathscr{E}$ is hereditary (the situation which will be explored in Section 8 ).

\section{StRONGLY GORENSTEIN SCHEMES}

In this section we only consider noetherian non-commutative schemes.

Definition 6.1. Let $(X, \mathscr{A})$ be a noetherian non-commutative scheme. We call it strongly Gorenstein if $X$ is equidimensional, $\mathscr{A}$ is Cohen-Macaulay as $\mathscr{O}_{X}$-module and inj. $\operatorname{dim}_{\mathscr{A}} \mathscr{A}=\operatorname{dim} X$.

Recall that an $\mathscr{A}$-module $\mathscr{M}$ is injective if and only if $\mathscr{A}_{x}$-modules $\mathscr{M}_{x}$ are injective for all $x \in X_{\mathrm{cl}}$ (the proof from (Hartshorne, 1966, Proposition 7.17) remains valid in non-commutative situation too). We need some basic facts about injective dimension for non-commutative rings. Now $R$ denotes a noetherian commutative local ring with the maximal ideal $\mathfrak{m}$ and the residue field $\mathbb{k}=R / \mathfrak{m}, A$ denotes an $R$ algebra finitely generated as $R$-module. Let also $\mathfrak{r}=\operatorname{rad} A$ and $\bar{A}=A / \mathfrak{r}$. As usually, for every ideal $I \subseteq R$ we denote by $V(I)$ the set of prime ideals containing $I$.

Theorem 6.2. inj.dim $M=\sup \left\{i \mid \operatorname{Ext}_{A}^{i}(\bar{A}, M) \neq 0\right\}$.

Just as in (Bruns and Herzog, 1993, Proposition 3.1.14), this theorem is an immediate consequence of the following lemma.

Lemma 6.3. Let $\mathfrak{p} \neq \mathfrak{m}$ be a prime ideal of $R, M$ be a noetherian $R$-module. Suppose that $\operatorname{Ext}_{A}^{i}(N, M)=0$ for any noetherian A-module $N$ such that $V\left(\operatorname{ann}_{R} N\right) \subset$

\footnotetext{
${ }^{6}$ We do not know whether the last condition implies the Cohen-Macaulay property, as it is in the commutative case.
} 
$V(\mathfrak{p})$ and $i>m$. Then also $\operatorname{Ext}_{A}^{i}(N, M)=0$ for any noetherian A-module $N$ such that $V\left(\operatorname{ann}_{R} N\right)=V(\mathfrak{p})$ and $i>m$.

Proof. Suppose that the condition is satisfied and let $V\left(\operatorname{ann}_{R} N\right)=V(\mathfrak{p})$. If $\mathfrak{q} \in$ Ass $N$ and $\mathfrak{q} \neq \mathfrak{p}$, there is a submodule $N^{\prime} \subseteq N$ such that $\mathfrak{q} N^{\prime}=0$. Therefore, $\operatorname{Ext}_{A}^{i}\left(N^{\prime}, M\right)=0$ for $i>m$ and we only have to prove that $\operatorname{Ext}_{A}^{i}\left(N / N^{\prime}, M\right)=0$ for $i>m$. Thus we can suppose that Ass $N=\{\mathfrak{p}\}$. Let $a \in \mathfrak{m} \backslash \mathfrak{p}$. Then $a$ is non-zerodivisor on $N$, i.e. we have the exact sequence $0 \rightarrow N \stackrel{a}{\rightarrow} N \rightarrow N / a N \rightarrow 0$. It gives and exact sequence

$$
\operatorname{Ext}_{A}^{i}(N, M) \stackrel{a}{\rightarrow} \operatorname{Ext}_{A}^{i}(N, M) \rightarrow \operatorname{Ext}_{A}^{i+1}(N / a N, M) .
$$

Obviously, $\operatorname{ann}_{R} N / a N \supset \mathfrak{p}$, so the last term is 0 if $i>m$. Therefore, $a \operatorname{Ext}_{A}^{i}(N, M)=$ $\operatorname{Ext}_{A}^{i}(N, M)$ and $\operatorname{Ext}_{A}^{i}(N, M)=0$ by Nakayama's Lemma.

Corollary 6.4. Let $\mathscr{M}$ be a coherent $\mathscr{A}$-module. Then

$$
\begin{aligned}
\operatorname{inj} \cdot \operatorname{dim}_{\mathscr{A}} \mathscr{M} & =\sup \left\{i \mid \operatorname{Ext}_{\mathscr{A}}^{i}(\mathscr{A}(x), \mathscr{M}) \neq 0 \text { for some } x \in X_{\mathrm{cl}}\right\}= \\
& =\sup \left\{\operatorname{inj} \cdot \operatorname{dim}_{\mathscr{A}_{x}} \mathscr{M}_{x} \mid x \in X_{\mathrm{cl}}\right\} .
\end{aligned}
$$

Here $\mathscr{A}(x)$ denotes $\mathscr{A} \otimes_{\mathscr{O}_{X}} \mathbb{k}(x)$.

\section{Corollary 6.5.}

$$
\begin{aligned}
\operatorname{gl} \operatorname{dim} \mathscr{A} & =\sup \left\{\operatorname{pr} \cdot \operatorname{dim}_{\mathscr{A}} \mathscr{A}(x) \mid x \in X_{\mathrm{cl}}\right\}= \\
& =\sup \left\{i \mid \operatorname{Ext}_{\mathscr{A}}^{i}(\mathscr{A}(x), \mathscr{A}(x)) \neq 0 \text { for some } x \in X_{\mathrm{cl}}\right\}= \\
& =\sup \left\{\operatorname{gl} \cdot \operatorname{dim} \mathscr{A}_{x} \mid x \in X_{\mathrm{cl}}\right\} .
\end{aligned}
$$

Lemma 6.6. Let $M$ be a noetherian A-module. If an element $a \in R$ is non-zerodivisor both on $A$ and on $M$, then inj. $\operatorname{dim}_{A} M=\operatorname{inj} \cdot \operatorname{dim}_{A / a A} M / a M$.

Proof. It just repeats that of (Bruns and Herzog, 1993, Corollary 3.1.15).

Corollary 6.7. Let $\mathbf{a}=\left(a_{1}, a_{2}, \ldots, a_{m}\right)$ be an A-sequence in $\mathfrak{m}$. Then $A$ is strongly Gorenstein if and only if so is $A / \mathbf{a} A$.

Corollary 6.8. $\mathscr{A}$ is strongly Gorenstein if and only if so is $\mathscr{A}^{\mathrm{op}}$.

Proof. The claim is local, so we can replace $\mathscr{A}$ by $A$. Corollary 6.7 reduces the proof to the case when $\operatorname{Kr} \cdot \operatorname{dim} R=0$, i.e. $A$ is just an artinian algebra. Then it is well-known (Auslander et al., 1997, Proposition IV.3.1).

For a noetherian non-commutative scheme $(X, \mathscr{A})$ we denote by $\mathrm{CM} \mathscr{A}$ the full subcategory of $\mathscr{A}$-mod consisting of such modules $\mathscr{M}$ that $\mathscr{M}_{x}$ is a maximal Cohen-Macaulay module over $\mathscr{O}_{X, x}$ for every point $x \in X$. The following results can be proved just as in the commutative case (see (Bruns and Herzog, 1993, Section 3.3)).

Theorem 6.9. Let $(X, \mathscr{A})$ be a strongly Gorenstein non-commutative scheme and $\mathscr{M} \in \mathrm{CM} \mathscr{A}$.

(1) $\mathscr{E} x t_{\mathscr{A}}^{i}(\mathscr{M}, \mathscr{A})=0$ for $i \neq 0$. 
(2) The natural map $\mathscr{M} \rightarrow \mathscr{H}^{\circ} m_{\mathscr{A}}\left(\mathscr{H}_{0} m_{\mathscr{A}}(\mathscr{M}, \mathscr{A}), \mathscr{A}\right)$ is an isomorphism. Thus the functor ${ }^{*}: \mathscr{M} \mapsto \mathscr{M}^{*}=\mathscr{H}_{\text {om }_{\mathscr{A}}}(\mathscr{M}, \mathscr{A})$ gives an exact duality between the categories $\mathrm{CM} \mathscr{A}$ and $\mathrm{CM} \mathscr{A}^{\mathrm{op}}$.

Let now $(X, \mathscr{A})$ be a strongly Gorenstein non-commutative scheme, $\mathscr{F} \in \mathrm{CM} \mathscr{A}$. Consider the endomorphism construction described in Example 4.7 Theorem 6.9 implies that the natural map $\phi(\mathscr{M}): \mathrm{F}_{\mathscr{F} \mathscr{M}} \rightarrow \mathrm{H}_{\mathscr{F} \mathscr{M}}$ is an isomorphism for $\mathscr{M}=$ $\mathscr{A}$, hence an isomorphism for any $\mathscr{M} \in \operatorname{lp} \mathscr{A}$.

Theorem 6.10. Let $(X, \mathscr{A})$ be strongly Gorenstein and contain enough locally projective modules, $\mathscr{F} \in \mathrm{CM} \mathscr{A}$. Then the restrictions of the functors $\mathrm{LF}_{\mathscr{F}}$ and $\mathrm{RH}_{\mathscr{F}}$ onto the subcategory $\mathscr{D}^{\mathrm{c}} \mathscr{A}$ are isomorphic. Thus the restriction of $\mathrm{LF} \mathscr{F}_{\text {onto }}$ $\mathscr{D}^{\mathrm{c}} \mathscr{A}$ is both left and right adjoint to the bilocalization functor $\mathrm{DG}_{\mathscr{F}}$.

Proof. As $\mathscr{A}$ has enough locally projective modules, any complex from $\mathscr{D}^{\mathrm{c}} \mathscr{A}$ is quasi-isomorphic to a finite complex $\mathscr{C} \bullet$ such that all $\mathscr{C}^{i}$ are from Ip $\mathscr{A}$. Then $\mathrm{LF}_{\mathscr{F}} \mathscr{C} \bullet=\mathrm{F}_{\mathscr{F}} \mathscr{C} \bullet$. On the other hand, by Theorem 6.9. $\mathrm{R}^{k} \mathrm{H}_{\mathscr{F}} \mathscr{C}^{i}=\mathscr{E} x t_{\mathscr{A}}^{k}\left(\mathscr{P}_{\mathscr{F}}, \mathscr{C}^{i}\right)=$ 0 for $k \neq 0$. Therefore, $\mathrm{RH}_{\mathscr{F}} \mathscr{C} \bullet=\mathrm{H}_{\mathscr{F}} \mathscr{C} \bullet \simeq \mathrm{F}_{\mathscr{F}} \mathscr{C} \bullet$.

\section{NON-COMMUTATIVE CURVES}

\subsection{Generalities.}

Definition 7.1. A non-commutative curve is a reduced non-commutative scheme $(X, \mathscr{A})$ such that $X$ is an excellent curve (equidimensional reduced noetherian scheme of dimension 1) and $\mathscr{A}$ is coherent and torsion free as $\mathscr{O}_{X}$-module.

As $X$ is excellent, then $\hat{\mathscr{A}}_{x}$, the $\mathfrak{m}_{x}$-adic completion of $\mathscr{A}_{x}$, is also reduced (has no nilpotent ideals). Therefore, for the local study of non-commutative curves we can use the usual results from the books (Curtis and Reiner, 1981; Reiner, 2003). We denote by $\mathscr{K}=\mathscr{K}(X)$ the sheaf of full rings of fractions of $\mathscr{O}_{X}$ and write $\mathscr{K} \mathscr{M}$ instead of $\mathscr{K} \otimes_{\mathscr{O}_{X}} \mathscr{M}$ for any $\mathscr{O}_{X}$-module $\mathscr{M}$. In particular, $\mathscr{K} \mathscr{A}$ is a $\mathscr{K}$ algebra. The sheaves $\mathscr{K} \mathscr{M}$ are locally constant; the stalks of $\mathscr{K}$ and $\mathscr{K} \mathscr{A}$ are semi-simple rings. The torsion part tors $\mathscr{M}$ of $\mathscr{M}$ is defined as the kernel of the natural map $\mathscr{M} \rightarrow \mathscr{K} \mathscr{M}$. We say that a coherent $\mathscr{A}$-module $\mathscr{M}$ is torsion free if tors $\mathscr{M}=0$, and we say that $\mathscr{M}$ is torsion if $\mathscr{K} \mathscr{M}=0$. Note that tors $\mathscr{M}$ is torsion and $\mathscr{M} /$ tors $\mathscr{M}$ is torsion free. We denote by tors $\mathscr{A}$ and tf $\mathscr{A}$ respectively the full subcategories of $\mathscr{A}$-mod consisting of torsion and of torsion free modules. We always consider a torsion free module $\mathscr{M}$ as a submodule of $\mathscr{K} \mathscr{M}$. In particular, we identify $\mathscr{M}_{x}$ with its natural image in $\mathscr{K} \mathscr{M}_{x}$. Note that for every submodule $\mathscr{N} \subseteq \mathscr{K} \mathscr{M}$ there is a natural embedding $\mathscr{K} \mathscr{N} \rightarrow \mathscr{K} \mathscr{M}$ and we identify $\mathscr{K} \mathscr{N}$ with the image of this embedding. A non-commutative curve $\left(X, \mathscr{A}^{\prime}\right)$ is said to be an over-ring of a non-commutative curve $(X, \mathscr{A})$ if $\mathscr{A} \subseteq \mathscr{A}^{\prime} \subset \mathscr{K} \mathscr{A}$. Then $\mathscr{A}^{\prime}$ is naturally considered as a coherent $\mathscr{A}$-module. The non-commutative curve $(X, \mathscr{A})$ is said to be normal if it has no proper over-rings. Since $X$ is excellent and $\mathscr{A}$ is reduced, the set $\left\{x \in X \mid \mathscr{A}_{x}\right.$ is not normal $\}$ is finite. Then it follows from (Drozd, 1985) that the set of over-rings of $\mathscr{A}$ satisfies the maximality condition: there are no infinite strictly ascending chains of over-rings of $\mathscr{A}$. 
Coherent torsion free $\mathscr{A}$-modules, in particular, over-rings of $\mathscr{A}$ can be constructed locally.

Lemma 7.2. Let $\mathscr{M}$ be a torsion free coherent $\mathscr{A}$-module.

(1) If $\mathscr{N}$ is a coherent $\mathscr{A}$-submodule of $\mathscr{K} \mathscr{M}$ such that $\mathscr{K} \mathscr{N}=\mathscr{K} \mathscr{M}$, then $\mathscr{N}_{x}=\mathscr{M}_{x}$ for almost all $x \in X$.

(2) Let $S \subset X_{\mathrm{cl}}$ be a finite set and for every $x \in S$ a finitely generated $\mathscr{A}_{x}$ submodule $N_{x} \subset \mathscr{K} \mathscr{M}_{x}$ is given such that $\mathscr{K} N_{x}=\mathscr{K} \mathscr{M}_{x}$. Then there is a unique $\mathscr{A}$-submodule $\mathscr{N} \subset \mathscr{K} \mathscr{M}$ such that $\mathscr{N}_{x}=N_{x}$ for every $x \in S$ and $\mathscr{N}_{x}=\mathscr{M}_{x}$ for all $x \notin S$.

(3) If $\mathscr{M}=\mathscr{A}$ and all $N_{x}$ in the preceding item are rings, then $\mathscr{N}$ is a subalgebra of $\mathscr{K} \mathscr{A}$, so $(X, \mathscr{N})$ is also a non-commutative curve and if $N_{x} \supseteq \mathscr{A}_{x}$ for all $x \in S,(X, \mathscr{N})$ is an over-ring of $(X, \mathscr{A})$.

Proof. We can suppose that $X$ is affine. Then the proof just repeats that of (Bourbaki, 1975, Ch. VII, $\S 3$, Theorem 3) with slight and obvious changes.

Lemma 7.3. Any non-commutative curve $(X, \mathscr{A})$ has enough invertible modules. Namely, the set

$$
\mathbf{L}_{\mathscr{A}}=\left\{\mathscr{A} \otimes_{\mathscr{O}_{X}} \mathscr{L} \mid \mathscr{L} \text { is an invertible } \mathscr{O}_{X} \text {-module }\right\}
$$

generates Qcoh $\mathscr{A}$ (hence, generates $\mathscr{D} \mathscr{A})$.

Proof. We must show that if $\mathscr{M}^{\prime} \subset \mathscr{M}$ is a proper submodule, there is a homomorphism $f: \mathscr{L} \rightarrow \mathscr{M}$ such that $\operatorname{Im} f \nsubseteq \mathscr{M}^{\prime}$. As $\operatorname{Hom}_{\mathscr{A}}\left(\mathscr{A} \otimes_{\mathscr{O}_{X}} \mathscr{L}, \mathscr{M}\right) \simeq$ $\operatorname{Hom}_{\mathscr{O}_{X}}(\mathscr{L}, \mathscr{M})$, we can suppose that $\mathscr{A}=\mathscr{O}_{X}$. Moreover, as every $\mathscr{A}$-module is a direct limit of its coherent submodules, we can suppose that $\mathscr{M}$ is coherent. Let first $\mathscr{M}^{\prime} \nsupseteq$ tors $\mathscr{M}$. Choose $x \in X_{\mathrm{cl}}$ such that tors $\mathscr{M}_{x} \nsubseteq \mathscr{M}_{x}^{\prime}$ and let $u_{x} \in$ tors $\mathscr{M}_{x} \backslash \mathscr{M}_{x}^{\prime}$. There is a global section $u \in \Gamma(X$,tors $\mathscr{M}) \subseteq \Gamma(X, \mathscr{M})$ such that $u_{x}$ is its image in $\mathscr{M}_{x}$. Then there is a homomorphism $f: \mathscr{O}_{X} \rightarrow \mathscr{M}$ such that $f l=u$, so $\operatorname{Im} f \nsubseteq \mathscr{M}^{\prime}$.

Let now $\mathscr{M}^{\prime} \supseteq$ tors $\mathscr{M}$. Since $\operatorname{Ext}_{\mathscr{O}_{X}}^{1}(\mathscr{L}$, tors $\mathscr{M})=0$ for any locally projective module $\mathscr{L}$, the map $\operatorname{Hom}_{\mathscr{O}_{X}}(\mathscr{L}, \mathscr{M}) \rightarrow \operatorname{Hom}_{\mathscr{O}_{X}}(\mathscr{L}, \mathscr{M} /$ tors $\mathscr{M})$ is surjective. Hence, we can suppose that $\mathscr{M}$ is torsion free. Let $\mathscr{M}_{y} \neq \mathscr{M}_{y}^{\prime}$ for some $y \in X_{\mathrm{cl}}$ and $u_{y} \in \mathscr{M}_{y} \backslash \mathscr{M}_{y}^{\prime}$. There is a homomorphism $\varphi: \mathscr{K} \rightarrow \mathscr{K} \mathscr{M}$ such that $\varphi 1=u_{y}$. Let $\mathscr{N}=\varphi\left(\mathscr{O}_{X}\right)$. The set $S=\left\{x \in X_{\mathrm{cl}} \mid \mathscr{N}_{x} \nsubseteq \mathscr{M}_{x}\right\}$ is finite; moreover, $y \notin S$. For every $x \in S$ there is an ideal $L_{x} \subseteq \mathscr{O}_{X, x}$ such that $L_{x} \simeq \mathscr{O}_{X, x}$ and $\varphi\left(L_{x}\right) \subseteq \mathscr{M}_{x}$. By Lemma 7.2 there is an ideal $\mathscr{L} \subseteq \mathscr{O}_{X}$ such that $\mathscr{L}_{x}=L_{x}$ for $x \in S$ and $\mathscr{L}_{x}=\mathscr{O}_{X, x}$ otherwise. It is an invertible ideal, $\varphi(\mathscr{L}) \subseteq \mathscr{M}$ and $\varphi(\mathscr{L}) \nsubseteq \mathscr{M}^{\prime}$.

We will use the duality for left and right coherent torsion free $\mathscr{A}$-modules established in the following theorem.

Theorem 7.4. (1) There is a canonical $\mathscr{A}$-module, i.e. such a module $\omega_{\mathscr{A}} \in$ tf $\mathscr{A}$ that inj. $\operatorname{dim}_{\mathscr{A}} \omega_{\mathscr{A}}=1$ and $\mathscr{E}$ nd $d_{\mathscr{A}} \omega_{\mathscr{A}} \simeq \mathscr{A}^{\mathrm{op}}$ (so $\omega_{\mathscr{A}}$ can be considered as an $\mathscr{A}$-bimodule). Moreover, $\omega_{\mathscr{A}}$ is isomorphic as a bimodule to an ideal of $\mathscr{A}$. 
We denote by $\mathscr{M}^{*}$, where $\mathscr{M} \in \mathscr{A}$-Mod (or $\mathscr{M} \in \mathscr{A}^{\text {op }}$-Mod) the $\mathscr{A}^{\text {op }}$ module (respectively, $\mathscr{A}$-module) $\mathscr{H}_{0} m_{\mathscr{A}}\left(\mathscr{M}, \omega_{\mathscr{A}}\right)$ (respectively, $\mathscr{H}_{\text {om }}{ }_{\mathscr{A}}$ op $\left.\left(\mathscr{M}, \omega_{\mathscr{A}}\right)\right)$.

(2) The natural map $\mathscr{M} \rightarrow \mathscr{M}^{* *}$ is an isomorphism for every $\mathscr{M} \in \mathrm{tf} \mathscr{A}$ (or $\mathscr{M} \in \mathrm{tf} \mathscr{A}^{\mathrm{op}}$ ) and the functors $\mathscr{M} \mapsto \mathscr{M}^{*}$ establish an exact duality of the categories tf $\mathscr{A}$ and $\mathrm{tf} \mathscr{A}^{\mathrm{op}}$. Moreover, if $\mathscr{M} \in \mathscr{A}$-mod, then $\mathscr{M}^{* *} \simeq$ $\mathscr{M} /$ tors $\mathscr{M}$.

Proof. Each local ring $\mathscr{O}_{x}=\mathscr{O}_{X, x}$ is excellent, so its integral closure in $\mathscr{K}_{x}$ is finitely generated and its completion $\hat{\mathscr{O}}_{x}$ is reduced. Therefore $\mathscr{O}_{x}$ has a canonical module $\omega_{x}$ which can be considered as an ideal in $\mathscr{O}_{x}$ (Herzog and Kunz, 1971, Korollar 2.12). Moreover, $\mathscr{O}_{x}$ is normal for almost all $x \in X_{\mathrm{cl}}$ and in this case we can take $\omega_{x}=\mathscr{O}_{X, x}$. By Lemma 7.2, there is an ideal $\omega_{X} \subseteq \mathscr{O}_{X}$ such that $\omega_{X, x}=\omega_{x}$ for each $x \in X$. Then inj. $\operatorname{dim}_{\mathscr{O}_{X}} \omega_{X}=\sup \left\{\operatorname{inj} \cdot \operatorname{dim}_{\mathscr{O}_{X, x}} \omega_{x}\right\}=1$. As the natural map $\mathscr{O}_{X, x} \rightarrow \operatorname{End}_{\mathscr{O}_{X, X}} \omega_{x}$ is an isomorphism for each $x \in X$, the natural map $\mathscr{O}_{X} \rightarrow \mathscr{E} n d_{\mathscr{O}_{X}} \omega_{X}$ is an isomorphism too. Therefore, $\omega_{X}$ is a canonical $\mathscr{O}_{X}$-module. Then it is known that the functor $\mathscr{M} \mapsto \mathscr{M}^{*}=\mathscr{H}_{0} m_{\mathscr{O}_{X}}\left(\mathscr{M}, \omega_{X}\right)$ is an exact selfduality of tf $\mathscr{O}_{X}$ and the natural map $\mathscr{M} \rightarrow \mathscr{M}^{* *}$ is an isomorphism. Set now $\omega_{\mathscr{A}}=\mathscr{H}_{0} \mathrm{~m}_{\mathscr{O}_{X}}\left(\mathscr{A}, \omega_{X}\right)$. Then $\mathscr{H}_{\text {om }}\left(\mathscr{M}, \omega_{\mathscr{A}}\right) \simeq \mathscr{H}_{\text {om }} \mathscr{O}_{X}\left(\mathscr{M}, \omega_{X}\right)$ for any $\mathscr{A}-$ module $\mathscr{M}$, whence all statements of the theorem follow.

As usually, we say that two non-commutative schemes $(X, \mathscr{A})$ and $(Y, \mathscr{B})$ are Morita equivalent if their categories of quasi-coherent modules are equivalent. A coherent locally projective $\mathscr{A}$-module $\mathscr{P}$ is said to be a local progenerator if $\mathscr{P}_{x}$ is a progenerator for $\mathscr{A}_{x}$ for all $x \in X$, that is $\mathscr{P}_{x}$ is projective over $\mathscr{A}_{x}$ and there is a surjection $r \mathscr{P}_{X} \rightarrow \mathscr{A}_{x}$ for some $r$. It follows from Theorem 4.3 that then $(X, \mathscr{A})$ is Morita equivalent to $(X, \mathscr{E})$, where $\mathscr{E}=\left(\mathscr{E} n d_{\mathscr{A}} \mathscr{P}\right)^{\text {op }}$.

Theorem 7.5. (1) Let $(X, \mathscr{A})$ and $(X, \mathscr{B})$ are two non-commutative curves such that $\mathscr{A}_{x}$ is Morita equivalent to $\mathscr{B}_{x}$ for every $x \in X_{\mathrm{cl}}$. Then $(X, \mathscr{A})$ and $(X, \mathscr{B})$ are Morita equivalent.

(2) Let now $(X, \mathscr{A})$ and $(Y, \mathscr{B})$ be two central non-commutative curves finite over a field. If they are Morita equivalent, there is an isomorpism $\tau: X \stackrel{\sim}{\rightarrow} Y$ such that, for every points $x \in X$ and $y=\tau(x)$, the rings $\left(\tau^{*} \mathscr{B}\right)_{x}$ and $\mathscr{A}_{x}$ are Morita equivalent.

Proof. 1 If $\mathscr{A}_{x}$ and $\mathscr{B}_{x}$ are Morita equivalent, there is a progenerator $P_{x}$ for $\mathscr{A}_{x}$ such that $\mathscr{B}_{x} \simeq\left(\operatorname{End}_{\mathscr{A}_{x}} P_{x}\right)^{\text {op }}$. There is a $\mathscr{K} \mathscr{A}$-module $\mathscr{V}$ such that $\mathscr{V} \simeq \mathscr{K} P_{x}$ for all $x \in X_{\mathrm{cl}}$. Choose a normal over-ring $\mathscr{A}^{\prime}$ of $\mathscr{A}$ and a coherent $\mathscr{A}^{\prime}$-submodule $\mathscr{M} \subset \mathscr{V}$ such that $\mathscr{K} \mathscr{M}=\mathscr{V}$. Then $\mathscr{M}$ is a local progenerator for $\mathscr{A}^{\prime}$. Set $\mathscr{B}^{\prime}=$ $\left(\mathscr{E} n d_{\mathscr{A}^{\prime}} \mathscr{M}\right)^{\text {op }}$ and $S=\left\{x \in X_{\mathrm{cl}} \mid \mathscr{A}_{x} \neq \mathscr{A}_{x}^{\prime}\right.$ or $\left.\mathscr{B}_{x} \neq \mathscr{B}_{x}^{\prime}\right\}$. The set $S$ is finite, so there is an $\mathscr{A}$-submodule $\mathscr{P} \subset \mathscr{V}$ such that $\mathscr{P}_{x}=P_{x}$ for $x \in S$ and $\mathscr{P}_{x}=\mathscr{M}_{x}$ for $x \notin S$. Then $\mathscr{P}$ is a local progenerator for $\mathscr{A}$ and $\mathscr{B} \simeq\left(\mathscr{E} n d_{\mathscr{A}} \mathscr{P}\right)^{\mathrm{op}}$.

2 follows from (Artin and Zhang, 1994, Section 6).

7.2. Hereditary non-commutative curves. We call a noetherian non-commutative scheme $(X, \mathscr{A})$ hereditary if all localizations $\mathscr{A}_{x}$ are hereditary rings, i.e. 
gl.dim $\mathscr{A}_{x}=1$. Then gl.dim $\mathscr{A}=1$ too, so $\operatorname{Ext}_{\mathscr{A}}^{2}(\mathscr{M}, \mathscr{N})=0$ for all $\mathscr{A}$-modules $\mathscr{M}, \mathscr{N}$. Suppose that $(X, \mathscr{A})$ is a hereditary non-commutative curve. Then any torsion free coherent $\mathscr{A}$-module $\mathscr{M}$ is locally projective, so $\mathscr{E} x t_{\mathscr{A}}^{1}(\mathscr{M}, \mathscr{N})=0$ for any $\mathscr{A}$-module $\mathscr{N}$. If $\mathscr{N}$ is coherent and torsion, it implies that $\operatorname{Ext}_{\mathscr{A}}^{1}(\mathscr{M}, \mathscr{N})=$ 0 . Therefore, every coherent $\mathscr{A}$-modules $\mathscr{M}$ splits as $\mathscr{M}=$ tors $\mathscr{M} \oplus \mathscr{M}^{\prime}$, where $\mathscr{M}^{\prime}$ is torsion free, hence locally projective. If a central non-commutative curve $(X, \mathscr{H})$ is hereditary, then $X$ is smooth. There is an effective description of hereditary non-commutative curves up to Morita equivalence.

First consider the case when $X=\operatorname{Spec} O$, where $O$ is a complete discrete valuation ring with the field of fractions $K$, the maximal ideal $\mathfrak{m}$ and the residue field $\mathbb{k}=O / \mathfrak{m}$. Let $H$ be a hereditary reduced $O$-algebra which is torsion free as $O$ module. Then $K H \simeq \operatorname{Mat}(n, D)$, where $D$ is a finite dimensional division algebra over $K$. There is a unique maximal $O$-order $\Delta \subset D$ (Reiner, 2003, Theorem 12.8). It contains a unique maximal ideal $\mathfrak{M}$, which is both left and right principal ideal. Let $n=\sum_{i=1}^{k} n_{i}$ for some positive integers $n_{i}, \mathbf{n}=\left(n_{1}, n_{2}, \ldots, n_{k}\right)$ and $H(\mathbf{n}, D)$ be the subring of $\operatorname{Mat}(n, \Delta)$ consisting of $k \times k$ block matrices $\left(A_{i j}\right)$ such that $A_{i j}$ is of size $n_{i} \times n_{j}$ and if $j>i$ all coefficients of $A_{i j}$ are from $\mathfrak{M}$. Let also $L=\Delta^{n}$ considered as $H(\mathbf{n}, D)$-module and $L_{i}$ be the submodule in $L$ consisting of such vectors $\left(\alpha_{1}, \alpha_{2}, \ldots, \alpha_{n}\right)$ that $\alpha_{j} \in \mathfrak{M}$ for $j \leq \sum_{q=1}^{i} n_{q}$. In particular, $L_{0}=L$ and $L_{k}=\mathfrak{M}^{n} \simeq L$. If necessary, we denote $L_{i}=L_{i}(H)$.

Theorem 7.6. (Reiner, 2003, Theorem 39.14) Let $O$ be a complete discrete valuation ring.

(1) Every connected hereditary O-order is isomorphic to $H(\mathbf{n}, D)$ for some tuple $\mathbf{n}=\left(n_{1}, n_{2}, \ldots, n_{k}\right)$, which is uniquely determined up to a cyclic permutation.

(2) Hereditary orders $H(\mathbf{n}, D)$ and $H\left(\mathbf{n}^{\prime}, D^{\prime}\right)$ are Morita equivalent if and only if $D \simeq D^{\prime}$ and $\mathbf{n}$ and $\mathbf{n}^{\prime}$ are of the same length.

(3) $L_{i}(0 \leq i<k)$ are all indecomposable projective $H(\mathbf{n}, D)$-modules and $U_{i}=$ $L_{i} / L_{i+1}$ are all simple $H(\mathbf{n}, D)$-modules (up to isomorphism).

Let now $(X, \mathscr{H})$ be a connected central hereditary non-commutative curve. Then $\mathscr{K} \mathscr{H}$ is a central simple $\mathscr{K}$-algebra: $\mathscr{K} \mathscr{H}=\operatorname{Mat}(n, \mathscr{D})$, where $\mathscr{D}$ is a central division algebra. For every closed point $x \in X$ the completion $\hat{\mathscr{D}}_{x}$ is isomorphic to $\operatorname{Mat}\left(m_{x}, D_{x}\right)$ for some central division algebra $D_{x}$ over $\hat{\mathscr{K}}_{x}$ and some integer $m_{x}=m_{x}(\mathscr{D})$. Therefore, for every closed point $x \in X, \hat{\mathscr{H}}_{x}$ is isomorphic to $H\left(\mathbf{n}, D_{x}\right)$ for some $\mathbf{n}=\left(n_{1}, n_{2}, \ldots, n_{k}\right)$, where $\sum_{i=1}^{k} n_{i}=m_{x} n$. Thus Theorems 7.5 and 7.6 give the following result.

Theorem 7.7. A central hereditary non-commutative curve $(X, \mathscr{H})$ is determined up to Morita equivalence by a central division $\mathscr{K}$-algebra $\mathscr{D}$ and a function $\kappa$ : $X_{\mathrm{cl}} \rightarrow \mathbb{N}$ such that $\kappa(x)=1$ for almost all $x \in X_{\mathrm{cl}}$.

Actually, $\kappa(x)$ is the number of non-isomorphic simple $\mathscr{H}$-modules $\mathscr{U}$ such that $\operatorname{supp} \mathscr{U}=\{x\}$. 
Remark 7.8. Representatives of a class given by $\mathscr{D}$ and $\kappa$ can be obtained as follows. Choose an integer $n$ such that $\kappa(x) \leq n m_{x}(\mathscr{D})$ for all $x \in X_{\mathrm{cl}}$. There is an $\mathscr{O}_{x^{-}}$ order $H_{x}$ in $\operatorname{Mat}(n, \mathscr{D})$ such that $\hat{H}_{x}=H\left(\mathbf{n}_{x}, D_{x}\right)$ for some $\mathbf{n}_{x}=\left(n_{1, x}, n_{2, x}, \ldots, n_{\kappa(x), x}\right)$. Fix a normal non-commutative curve $(X, \Delta)$ such that $\mathscr{K} \Delta=\mathscr{D}$. Then we can define $\mathscr{H}=\mathscr{H}(\mathbf{n}, \mathscr{D})$ as the non-commutative curve such that $\mathscr{K} \mathscr{H}=\operatorname{Mat}(n, \mathscr{D})$, $\mathscr{H}_{x}=\operatorname{Mat}\left(n, \Delta_{x}\right)$ if $\kappa(x)=1$ and $\mathscr{H}_{x}=H_{x}$ if $\kappa(x)>1$.

Let $S=\{x \in X \mid \kappa(x)>1\}, \mathscr{L}=\Delta^{n}$ considered as $\mathscr{H}$-module. Consider the submodules $\mathscr{L}_{x, i}(0 \leq i \leq \kappa(x))$ such that $\left(\widehat{\mathscr{L}}_{x, i}\right)_{x}=L_{i}\left(\hat{H}_{x}\right)$ and $\left(\mathscr{L}_{x, i}\right)_{y}=\mathscr{L}_{y}$ if $y \neq x$. Let also $U_{x, i}=\mathscr{L}_{x, i} / \mathscr{L}_{x, i+1}(0 \leq i<\kappa(x))$. Then $U_{x, i}$ are all simple $\mathscr{H}$ modules (up to isomorphism). Note that $\mathscr{L}_{x, 0}=\mathscr{L}$ for every point $x$.

Theorem 7.9. Let $\mathscr{H}=\mathscr{H}(\mathbf{n}, \mathscr{D})$.

(1) The set

$$
\mathbb{L}_{\mathscr{H}}=\{\mathscr{L}\} \cup\left\{\mathscr{L}_{x, i} \mid x \in S, 1 \leq k \leq \kappa(x)\right\}
$$

classically generates $\mathscr{D} \mathfrak{H}$, hence generates $\mathscr{D} \mathscr{H}$ (see (Lunts, 2010, Theorem 2.2)).

(2) $\mathscr{D} \mathscr{H} \simeq \mathscr{D} \mathbb{A}$, where $\mathbb{A}$ denotes the DG-category with the set of objects $\mathbb{L}_{\mathscr{A}}$ and $\mathbb{A}\left(\mathscr{L}^{\prime}, \mathscr{L}^{\prime \prime}\right)=\operatorname{RHom}_{\mathscr{A}}\left(\mathscr{L}^{\prime}, \mathscr{L}^{\prime \prime}\right)$.

Proof. 1. Obviously, $\left\langle\mathbb{L}_{\mathscr{H}}\right\rangle_{\infty}$ contains all simple $\mathscr{H}$-modules. Therefore, it contains all torsion coherent $\mathscr{H}$-modules, as well as all coherent $\mathscr{H}$-submodules of $\mathscr{K} \mathscr{L}$. If $\mathscr{M}$ is a coherent torsion free $\mathscr{H}$-module, it contains a submodule $\mathscr{N}$ isomorphic to a submodule of $\mathscr{K} \mathscr{L}$ such that $\mathscr{M} / \mathscr{N}$ is also torsion free. It implies that $\left\langle\mathbb{L}_{\mathscr{H}}\right\rangle_{\infty}$ contains all coherent $\mathscr{H}$-modules, hence coincides with $\mathscr{D}^{\mathrm{c}} \mathscr{H}$.

2 follows now from (Lunts, 2010, Proposition 2.6).

Corollary 7.10. Let $\mathbb{k}$ be an algebraically closed field.

(1) A connected hereditary algebraic non-commutative curve over $\mathbb{k}_{\mathrm{k}}$ is defined up to Morita equivalence by a pair $(X, \kappa)$, where $X$ is a smooth connected algebraic curve over $\mathbb{k}$ and $\kappa: X_{\mathrm{cl}} \rightarrow \mathbb{N}$ is a function such that $\kappa(x)=1$ for almost all $x$. Representatives of the Morita class given by such a pair are $\mathscr{H}(\mathbf{n}, \mathscr{K})$ as described in Remark 7.8

(2) Two connected hereditary non-commutative curves given by the pairs $(X, \kappa)$ and $\left(X^{\prime}, \kappa^{\prime}\right)$ are Morita equivalent if and only if there is an isomorphism $\tau: X \rightarrow X^{\prime}$ such that $\kappa^{\prime}(\tau(x))=\kappa(x)$ for all $x \in X_{\mathrm{cl}}$.

In this case we write $\mathscr{H}(\mathbf{n}, X)$ instead of $\mathscr{H}(\mathbf{n}, \mathscr{K})$.

Proof. The Brauer group of $\mathscr{K}$ is trivial (Lang, 1952, Theorem 17). Therefore, the algebra $\mathscr{D}$ in Theorem 7.7 coincides with $\mathscr{K}$.

We say that a central non-commutative curve $(X, \mathscr{A})$ is rational (over a field $\mathbb{k}$ ) if all simple components of the algebra $\mathscr{K} \mathscr{A}$ are of the form $\operatorname{Mat}(n, \mathscr{K})$. Then the curve $X$ is also rational over $\mathbb{k}$.

Theorem 7.11. Let $(X, \mathscr{H})$ be a connected rational hereditary non-commutative curve over a field $\mathbb{k}$ and $\kappa: X_{\mathrm{cl}} \rightarrow \mathbb{N}$ be the corresponding function. Let $S=$ $\left\{x \in X_{\mathrm{cl}} \mid \kappa(x)>1\right\}, o \in X_{\mathrm{cl}}$ be an arbitrary point. 
(1) The set

$$
\overline{\mathbb{L}}_{\mathscr{H}}=\{\mathscr{L}, \mathscr{L}(-o)\} \cup\left\{\mathscr{L}_{x, i} \mid x \in S, 1 \leq i<\kappa(x)\right\}
$$

classically generates $\mathscr{D}^{\mathrm{c}} \mathscr{H}$, hence generates $\mathscr{D} \mathscr{H}$.

(2) If $\mathscr{L}^{\prime}, \mathscr{L}^{\prime \prime} \in \overline{\mathbb{L}}_{\mathscr{H}}$, then $\operatorname{Ext}_{\mathscr{H}}^{k}\left(\mathscr{L}^{\prime}, \mathscr{L}^{\prime \prime}\right)=0$ for all $k>0$, while

$$
\operatorname{dim}_{\operatorname{Hom}} \mathscr{H}\left(\mathscr{L}^{\prime}, \mathscr{L}^{\prime \prime}\right)= \begin{cases}1 & \text { if } \mathscr{L}^{\prime}=\mathscr{L}^{\prime \prime}, \\ & \text { or } \mathscr{L}^{\prime}=\mathscr{L}(-o), \mathscr{L}^{\prime \prime}=\mathscr{L}_{x, i}, \\ & \text { or } \mathscr{L}^{\prime}=\mathscr{L}_{x, i}, \mathscr{L}^{\prime \prime}=\mathscr{L}, \\ & \text { or } \mathscr{L}^{\prime}=\mathscr{L}_{x, j}, \mathscr{L}^{\prime \prime}=\mathscr{L}_{x, i}, j>i \\ 2 & \text { if } \mathscr{L}^{\prime}=\mathscr{L}(-o), \mathscr{L}^{\prime \prime}=\mathscr{L}, \\ 0 & \text { in all other cases. }\end{cases}
$$

In particular, $\overline{\mathbb{L}}_{\mathscr{H}}$ is a tilting set for the category $\mathscr{D} \mathscr{H}$.

(3) If $\theta_{x, i}$ are generators of the spaces $\operatorname{Hom}_{\mathscr{H}}\left(\mathscr{L}_{x, i}, \mathscr{L}_{x, i-1}\right)(1 \leq i \leq \kappa(x))$, then the products $\theta_{x}=\theta_{x, 1} \theta_{x, 2} \ldots \theta_{x, \kappa(x)}$ are non-zero and any two of them generate $\operatorname{Hom}_{\mathscr{H}}(\mathscr{L}(-o), \mathscr{L})$.

Proof. 1. If $X \simeq \mathbb{P}^{1}$, then all sheaves $\mathscr{O}(-x)$, hence all sheaves $\mathscr{L}(-x)$ are isomorphic. Moreover, in this case $\mathscr{L}_{x, \kappa(x)} \simeq \mathscr{L}(-x)$ for any $x \in X_{\mathrm{cl}}$, so we can apply Theorem 7.9

2. From the definition of $\mathscr{L}$ and $\mathscr{L}_{x, i}$ it immediately follows that

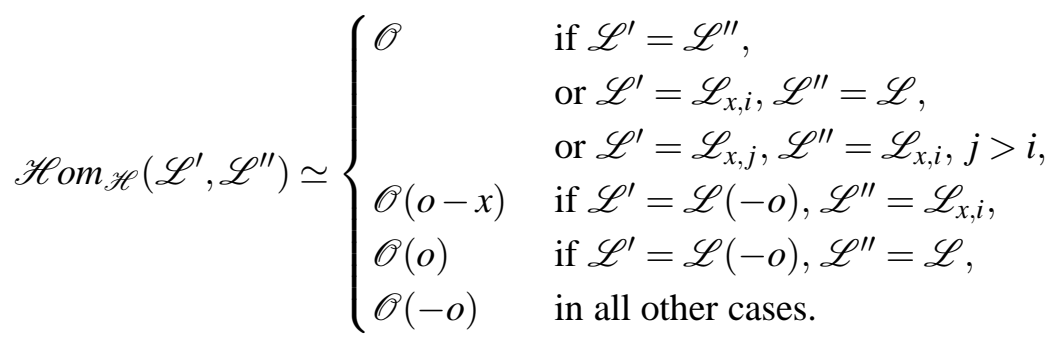

Since $\operatorname{Ext}_{\mathscr{H}}^{i}\left(\mathscr{L}^{\prime}, \mathscr{L}^{\prime \prime}\right)=H^{i}\left(\mathscr{H}_{0} m_{\mathscr{H}}\left(\mathscr{L}^{\prime}, \mathscr{L}^{\prime \prime}\right)\right)$, it implies the statement.

3. One easily sees that, if $x=(1: \xi)$ as the point of $\mathbb{P}^{1}$, then $\theta_{x}$, up to a scalar, is the multiplication by $t-\xi$, where $t$ is the affine coordinate on the affine chart $\mathbb{A}_{0}^{1}$. Now the statement is obvious.

Recall that a canonical algebra (Ringel, 1984, 3.7) is given by a sequence of integers $\left(k_{1}, k_{2}, \ldots, k_{r}\right)$, where $r \geq 2$ and all $k_{i} \geq 2$ if $r>2$, and a sequence $\left(\lambda_{3}, \lambda_{4}, \ldots, \lambda_{r}\right)$ of different non-zero elements from $\mathbb{k}_{k}$ (if $r=2$, this sequence is empty). Namely, this algebra, which we denote by $R\left(k_{1}, k_{2}, \ldots, k_{r} ; \lambda_{3}, \ldots, \lambda_{r}\right)$, is 
given by the quiver

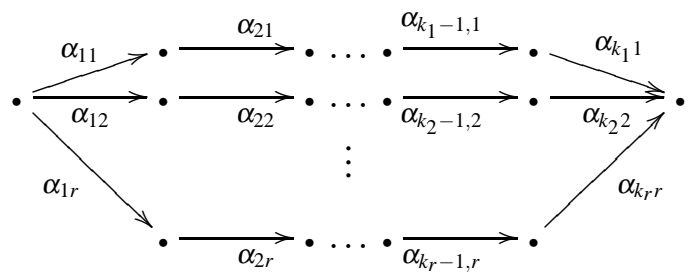

with relations $\alpha_{j}=\alpha_{1}+\lambda_{j} \alpha_{2}$ for $3 \leq j \leq r$, where $\alpha_{j}=\alpha_{k_{j} j} \ldots \alpha_{2 j} \alpha_{1 j}$. Certainly, if $r=2$, it is the path algebra of a quiver of type $\tilde{\mathrm{A}}_{k_{1}+k_{2}}$. In particular, if $r=2, k_{1}=$ $k_{2}=1$, it is the Kronecker algebra.

Corollary 7.12. Let $(X, \mathscr{H})$ be a rational projective hereditary non-commutative curve, $\kappa: X_{\mathrm{cl}} \rightarrow \mathbb{N}$ be the corresponding function. Let $\mathscr{T}=\bigoplus_{\mathscr{F} \in \mathbb{L}_{\mathscr{H}}} \mathscr{F}$ and $\Lambda=\left(\operatorname{End}_{\mathscr{H}} \mathscr{T}\right)^{\mathrm{op}}$. If $S=\left\{x_{1}, x_{2}, \ldots, x_{r}\right\}$ with $r \geq 2$, we set $k_{i}=\kappa\left(x_{i}\right)$. If $S=\{x\}$, we set $r=2, k_{2}=1$ and $k_{1}=\kappa(x)$. If $S=\emptyset$, we set $r=2, k_{1}=k_{2}=1$.

(1) $\mathscr{T}$ is a tilting $\mathscr{H}$-module, i.e. $\operatorname{Ext}_{\mathscr{H}}^{i}(\mathscr{T}, \mathscr{T})=0$ for $i \neq 0$ and $\mathscr{T}$ is a local progenerator for $\mathscr{H}$.

(2) $\Lambda \simeq R\left(k_{1}, k_{2}, \ldots, k_{r} ; \lambda_{3}, \ldots, \lambda_{r}\right)$ for some $\lambda_{3}, \ldots, \lambda_{r}$.

(3) The functor $\operatorname{Hom}_{\mathscr{H}}\left(\mathscr{T},_{-}\right)$induces an equivalence $\mathscr{D} \mathscr{H} \simeq \mathscr{D} \Lambda$.

Actually, the preceding considerations also show that a rational projective hereditary non-commutative curve is Morita equivalent to a weighted projective line (Geigle and Lenzing, 1987). It can also be deduced from the description of hereditary non-commutative curves and the remark on page 271 of (Geigle and Lenzing, 1987).

\section{KÖNIG RESOLUTION AND TILTING}

8.1. König resolution. For a non-commutative curve $(X, \mathscr{A})$ we denote by $\mathscr{J}=$ $\mathscr{J}(\mathscr{A})$ its ideal defined by the localizations as follows:

$$
\mathscr{J}_{x}= \begin{cases}\mathscr{A} & \text { if } \mathscr{A} \text { is hereditary } \\ \operatorname{rad} \mathscr{A} & \text { otherwise }\end{cases}
$$

We also denote by $\mathscr{A}^{\sharp}$ the non-commutative curve $\mathscr{E} n d_{\mathscr{A} \text { op }} \mathscr{J}$ (the endomorphism algebra of $\mathscr{J}$ as of right $\mathscr{A}$-module). It can and will be identified with an over-ring of $\mathscr{A}$. The following result is proved in (Reiner, 2003, Theorem 39.14).

Proposition 8.1. $\mathscr{A}=\mathscr{A}^{\sharp}$ if and only if $\mathscr{A}$ is hereditary.

Thus we can construct a chain of over-rings

$$
\mathscr{A}=\mathscr{A}_{1} \subset \mathscr{A}_{2} \subset \mathscr{A}_{3} \subset \ldots \subset \mathscr{A}_{n+1}=\mathscr{H},
$$

where $\mathscr{A}_{i+1}=\mathscr{A}_{i}^{\sharp}(1 \leq i \leq n)$ and $\mathscr{H}$ is hereditary. We call $n$ the level of $\mathscr{A}$. The non-commutative curve $\tilde{\mathscr{A}}=\left(\mathscr{E} n d_{\mathscr{A}} \mathscr{A}_{\oplus}\right)^{\text {op }}$, where $\mathscr{A}_{\oplus}=\bigoplus_{i=1}^{n+1} \mathscr{A}_{i}$ is called the 
König resolution of the non-commutative curve $\mathscr{A}$. It is identified with the algebra of matrices

$$
\tilde{\mathscr{A}}=\left(\begin{array}{ccccc}
\mathscr{A}_{11} & \mathscr{A}_{12} & \mathscr{A}_{13} & \ldots & \mathscr{A}_{1, n+1} \\
\mathscr{A}_{21} & \mathscr{A}_{22} & \mathscr{A}_{23} & \ldots & \mathscr{A}_{2, n+1} \\
\mathscr{A}_{31} & \mathscr{A}_{32} & \mathscr{A}_{33} & \ldots & \mathscr{A}_{3, n+1} \\
\ldots \ldots & \ldots & \ldots & \ldots \ldots & \ldots \\
\mathscr{A}_{n+1,1} & \mathscr{A}_{n+1,2} & \mathscr{A}_{n+1,3} & \ldots & \mathscr{A}_{n+1, n+1}
\end{array}\right)
$$

where $\mathscr{A}_{i j}=\mathscr{H}_{\text {om }}\left(\mathscr{A}_{i}, \mathscr{A}_{j}\right)$. Note that $\mathscr{A}_{i j}=\mathscr{A}_{j}$ if $i \leq j$, while $\mathscr{A}_{i+1, i} \supseteq \mathscr{J}\left(\mathscr{A}_{i}\right)$. Let $e_{i}(1 \leq i \leq n+1)$ be the diagonal idempotents of $\tilde{\mathscr{A}}, \mathscr{P}=\tilde{\mathscr{A}} e_{1}$ and $\tilde{\mathscr{P}}=$ $\tilde{\mathscr{A}} e_{n+1}$. Then $\left(\mathscr{E} n d_{\mathscr{A}} \mathscr{P}\right)^{\mathrm{op}} \simeq \mathscr{A}$ and $\left(\mathscr{E} n d_{\mathscr{A}} \tilde{\mathscr{P}}\right)^{\mathrm{op}} \simeq \mathscr{H}$, so both $\mathscr{A}$ and $\mathscr{H}$ are minors of $\tilde{A}$ and the categories $\mathscr{A}$-Mod and $\mathscr{H}-\operatorname{Mod}(\mathscr{D} \mathscr{A}$ and $\mathscr{D} \mathscr{H})$ are bilocalizations of $\tilde{\mathscr{A}}$-Mod (respectively, of $\mathscr{D} \mathscr{\mathscr { A }}$ ) with respect to bilocalization functors $\mathrm{G}=\mathscr{H}_{o m_{\tilde{A}}}\left(\mathscr{P},_{-}\right)$and $\tilde{\mathrm{G}}=\mathscr{H}$ om $m_{\mathscr{A}}\left(\tilde{\mathscr{P}},_{-}\right)$.

We also denote $\varepsilon_{k}=\sum_{j=k+1}^{n+1} e_{k}, \mathscr{I}_{k}=\tilde{\mathscr{A}} \varepsilon_{k} \tilde{\mathscr{A}}, \mathscr{Q}_{k}=\tilde{\mathscr{A}} / \mathscr{I}_{k}$ and $\mathscr{P}_{k}=\mathscr{Q}_{k} e_{k}$. The next result justifies the term "resolution" in the name of $\tilde{\mathscr{A}}$.

Theorem 8.2. $\mathbf{C}=\left(\tilde{\mathscr{A}}, \tilde{\mathscr{P}}, \mathscr{Q}_{n}, \mathscr{P}_{n}, \mathscr{Q}_{n-1}, \mathscr{P}_{n-1}, \ldots, \mathscr{Q}_{2}, \mathscr{P}_{2}, \mathscr{Q}_{1}\right)$ is a heredity relating chain between $\tilde{A}$ and $\mathscr{Q}_{1}=\mathscr{A} / \mathscr{A}_{21}$. Moreover, $\left(\mathscr{E} \text { nd }_{\mathscr{Q}_{i}} \mathscr{P}_{i}\right)^{\mathrm{op}}=\mathscr{A}_{i} / \mathscr{A}_{i+1, i}$ is semi-simple, so $\tilde{\mathscr{A}}$ is a quasi-hereditary non-commutative curve of level $n$ and $\operatorname{gl} \operatorname{dim} \tilde{\mathscr{A}} \leq 2 n$.

Proof. One easily verifies that $\mathscr{I}_{i}$ is the ideal of the matrices

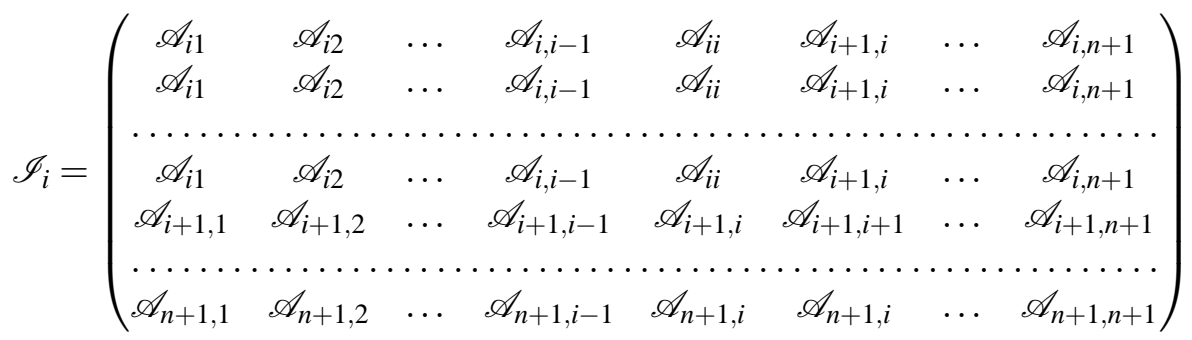

Therefore, $\mathscr{Q}_{i}$ is identified with the algebra of $i \times i$ matrices $\left(a_{k l}\right)$, where $a_{k l} \in$ $\mathscr{A}_{k l} / \mathscr{A}_{i+1, l}$. Thus $a_{i i} \in \mathscr{A}_{i} / \mathscr{A}_{i+1, i}$ and the latter algebra is semi-simple. Hence $\mathscr{E} n d_{\mathscr{Q}_{i}} \mathscr{P}_{i}=e_{i} \mathscr{Q}_{i} e_{i}=\mathscr{A}_{i} / \mathscr{A}_{i, i+1}$ is semi-simple. On the other hand, $\mathscr{I}_{\mathscr{P}_{i}}=\mathscr{Q}_{i} e_{i} \mathscr{Q}_{i}=$ $\mathscr{I}_{i+1} / \mathscr{I}_{i}$, whence $\mathscr{Q}_{i-1}=\mathscr{Q}_{i} / \mathscr{I}_{\mathscr{P}_{i}}$, so $\mathbf{C}$ is a relating chain. Moreover, $\mathscr{I}_{i}$ is projective as a right $\tilde{\mathscr{A}}$-module, hence $\mathscr{I}_{i} / \mathscr{I}_{i+1}$ is projective as a right $\mathscr{Q}_{i}$-module, so this relating chain is heredity. As $\mathscr{H}=\left(\mathscr{E} n d_{\tilde{\mathscr{A}}} \tilde{\mathscr{P}}\right)^{\text {op }}$ is hereditary, $\tilde{\mathscr{A}}$ is quasihereditary, and as all $\mathscr{E} n d_{\mathscr{Q}_{i}} \mathscr{P}_{i}$ are semi-simple, gl.dim $\tilde{\mathscr{A}} \leq 2 n$ by Corollary 5.3

It means that the functor DG: $\mathscr{D} \mathscr{A} \rightarrow \mathscr{D} \mathscr{A}$ defines a categorical resolution of $\mathscr{D} \mathscr{A}$ in the sense of (Lunts, 2010). If $\mathscr{A}$ is strongly Gorenstein, Theorem 6.10 shows that this resolution is weakly crepant, i.e. its left and right adjoints coincide on perfect complexes (small objects in $\mathscr{D} \mathscr{A}$ ).

We denote by $\overline{\mathscr{A}}_{i}$ the semi-simple algebra $\mathscr{A}_{i} / \mathscr{A}_{i+1, i} \simeq \mathscr{E} n d_{\mathscr{Q}_{i}} \mathscr{P}_{i}$. 
Corollary 8.3. The derived category $\mathscr{D} \tilde{\mathscr{A}}$ has two semi-orthogonal decompositions: $\mathscr{D} \mathscr{A}=\left\langle\mathscr{T}_{1}, \mathscr{T}_{2}, \ldots, \mathscr{T}_{n}, \mathscr{T}\right\rangle$ and $\mathscr{D} \mathscr{R}=\left\langle\mathscr{T}^{\prime}, \mathscr{T}_{n}^{\prime}, \ldots, \mathscr{T}_{2}^{\prime}, \mathscr{T}_{1}^{\prime}\right\rangle$, where $\mathscr{T} \simeq$ $\mathscr{T}^{\prime} \simeq \mathscr{D} \mathscr{H}$ and $\mathscr{T}_{i} \simeq \mathscr{T}_{i}^{\prime} \simeq \mathscr{D} \overline{\mathscr{A}}_{i}$.

Note that usually $\mathscr{T} \neq \mathscr{T}^{\prime}$ as well as $\mathscr{T}_{i} \neq \mathscr{T}_{i}^{\prime}$ for $i>1$, though $\mathscr{T}_{1}=\mathscr{T}_{1}^{\prime}=$ $\mathscr{D}\left(\tilde{\mathscr{A}} / \mathscr{I}_{2}\right)$ naturally embedded into $\mathscr{D} \tilde{\mathscr{A}}$.

Let $F$ and $\tilde{F}$ be, respecrively, the left adjoints of $G$ and $\tilde{G} ; H$ and $\tilde{H}$ be, respectively, the right adjoints of $G$ and $\tilde{G}$. Then we have the diagram of bilocalizations

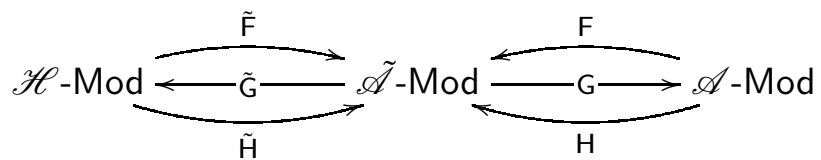

As $\mathscr{H}$ is an over-ring of $\mathscr{A}$, there is a morphism $v:(X, \mathscr{H}) \rightarrow(X, \mathscr{A})$. In the case of commutative curves, it is just the normalization of $X$. This morphism induces the direct image functor $v_{*}: \mathscr{H}$-Mod $\rightarrow \mathscr{A}$-Mod and its left and right adjoints, respectively, $v^{*}$ (the inverse image functor) and $v^{!}$. We show that they coincide (in some cases up to twist) with the compositions of the functors from the diagram (3).

Theorem 8.4. (1) $v_{*} \simeq \mathrm{G} \tilde{\mathrm{F}}$ and $v^{!} \simeq \tilde{\mathrm{G}} \mathrm{H}$.

(2) $\tilde{\mathrm{G}} \mathrm{F} \simeq \mathscr{C} \otimes \mathscr{H} v^{*}$ and $\mathrm{G} \tilde{\mathrm{H}} \simeq v_{*}\left(\mathscr{C}^{\prime} \otimes \mathscr{H}-\right)$, where $\mathscr{C}=\mathscr{H}_{\text {om }}(\mathscr{H}, \mathscr{A})$ is the conductor of $\mathscr{H}$ in $\mathscr{A}$ and $\mathscr{C}^{\prime}=\mathscr{H}$ om $_{\mathscr{H}}(\mathscr{C}, \mathscr{H})$ is its dual $\mathscr{H}$-module.

Proof. We prove the equalities 1 , the equalities 2 are proved analogously.

As $e_{1} \tilde{\mathscr{P}}=\mathscr{H}$ as an $\mathscr{A}$ - $\mathscr{H}$-bimodule,

$$
\mathrm{G} \tilde{\mathrm{F}}(\mathscr{M})=\mathscr{H}_{o m_{\mathscr{A}}}(\mathscr{P}, \tilde{P} \otimes \mathscr{H} \mathscr{M}) \simeq e_{1} \tilde{\mathscr{P}} \otimes \mathscr{H} \mathscr{M}=\mathscr{M}
$$

considered as an $\mathscr{A}$-module, and it is just $v_{*} \mathscr{M}$. On the other hand,

$$
\begin{aligned}
\tilde{\mathrm{G}} \mathrm{H}(\mathscr{N}) & =\mathscr{H}_{o m_{\mathscr{A}}}\left(\tilde{\mathscr{P}}, \mathscr{H}_{o m_{\mathscr{A}}}\left(\mathscr{P}^{\vee}, \mathscr{N}\right)\right) \simeq e_{n+1} \mathscr{H}_{o m_{\mathscr{A}}}\left(\mathscr{P}^{\vee}, \mathscr{N}\right) \\
& \simeq \mathscr{H} o m_{\mathscr{A}}\left(\mathscr{P}^{\vee} e_{n+1}, \mathscr{N}\right) \simeq \mathscr{H} o m_{\mathscr{A}}(\mathscr{H}, \mathscr{N})=v^{!} \mathscr{N} .
\end{aligned}
$$

8.2. Rational case: Tilting. Now we suppose that the non-commutative curve $(X, \mathscr{A})$ is rational over an algebraically closed field $\mathbb{k}$. We keep the notations of the preceding subsection. According to Corollary 7.12 , the hereditary algebra $\mathscr{H}$ has a tilting module $\mathscr{T}$ such that $\left(\operatorname{End}_{\mathscr{H}} \mathscr{T}\right)^{\mathrm{op}}=\Lambda$ is a Ringel canonical algebra. Set $\mathscr{T}^{\prime}=\tilde{\mathrm{F}}(\mathscr{T}), \mathscr{Q}=\mathscr{Q}_{n}$.

Theorem 8.5. (1) $\tilde{\mathscr{T}}=\mathscr{Q}[-1] \oplus \mathscr{T}^{\prime}$ is a tilting complex for $\tilde{\mathscr{A}}$, i.e. $\tilde{\mathscr{T}} \in$ $\mathscr{D}^{\mathrm{c}} \tilde{\mathscr{A}}, \operatorname{Hom}_{\mathscr{D} \mathscr{A}}(\tilde{\mathscr{T}}, \tilde{\mathscr{T}}[m])=0$ for $m \neq 0$ and $\tilde{\mathscr{T}}$ generates $\mathscr{D} \mathscr{A}$. Therefore, the functor $\mathrm{RHom}_{\mathscr{D} \tilde{\mathscr{A}}}\left(\tilde{\mathscr{T}},_{-}\right)$establishes an equivalence $\mathscr{D} \mathscr{A} \simeq \mathscr{D} \tilde{\Lambda}$, where $\tilde{\Lambda}=\left(\operatorname{End}_{\mathscr{D} \tilde{A}} \tilde{\mathscr{T}}\right)^{\mathrm{op}}$.

(2) The algebra $\tilde{\Lambda}$ is quasi-hereditary.

(3) $\operatorname{gl} \operatorname{dim} \tilde{\Lambda} \leq 2 n+1$. 
Proof. 1. As $\tilde{\mathscr{P}}$ is a projective right $\mathscr{H}$-module, $\widetilde{\mathrm{LF}}$ is an exact full embedding. It also maps perfect complexes to perfect complexes by Theorem 4.53. Therefore, $\mathscr{T}^{\prime} \in \mathscr{D}^{\mathrm{c}} \tilde{\mathscr{A}}, \operatorname{Hom}_{\mathscr{D} \mathscr{A}}\left(\mathscr{T}^{\prime}, \mathscr{T}^{\prime}[m]\right)=0$ for $m \neq 0$ and $\mathscr{T}^{\prime}$ generates Im $\widetilde{L F}$. On the other hand, $\mathscr{Q}$ generates $\operatorname{Ker} \tilde{G} \simeq \mathscr{Q}$-Mod. Since $\langle\operatorname{Ker} \widetilde{D G}, \operatorname{Im} \widetilde{L F}\rangle$ is a semiorthogonal decomposition of $\mathscr{D} \tilde{\mathscr{A}}, \tilde{\mathscr{T}}$ generates $\mathscr{D} \tilde{\mathscr{A}}$ and $\operatorname{Hom}_{\mathscr{D} \mathscr{\mathscr { A }}}\left(\mathscr{T}^{\prime}, \mathscr{Q}[m]\right)=$ 0 for all $m$. As $\operatorname{dimsupp} \mathscr{Q}=0, \operatorname{Ext}_{\mathscr{\mathscr { A }}}^{k}(\mathscr{Q}, \mathscr{M})=H^{0}\left(X, \mathscr{E} x t_{\mathscr{\mathcal { A }}}^{k}(\mathscr{Q}, \mathscr{M})\right)$ for every quasi-coherent $\tilde{\mathscr{A}}$-module $\mathscr{M}$. A locally projective resolution of $\mathscr{Q}$ is $0 \rightarrow$ $\mathscr{I}_{\tilde{P}} \rightarrow \tilde{\mathscr{A}} \rightarrow \mathscr{Q} \rightarrow 0$, hence $\mathscr{Q} \in \mathscr{D}^{\mathrm{c}} \tilde{\mathscr{A}}$ and $\operatorname{Ext}_{\tilde{\mathscr{A}}}^{k}(\mathscr{Q}, \mathscr{M})=0$ for $k>1$. Moreover, if $\mathscr{M}$ is a $\mathscr{Q}$-module, that is $\mathscr{I}_{\tilde{\mathscr{P}}} \mathscr{M}=0$, then $\mathscr{H}_{o m_{\tilde{\mathscr{I}}}}\left(\mathscr{I}_{\tilde{\mathscr{P}}}, \mathscr{M}\right)=0$, since $\mathscr{I}_{\tilde{\mathscr{P}}}=\mathscr{I}_{\tilde{\mathscr{P}}}^{2}$. Hence $\operatorname{Ext}_{\mathscr{\mathscr { A }}}^{1}(\mathscr{Q}, \mathscr{M})=0$. Evidently, $\operatorname{Hom}_{\tilde{\mathscr{A}}}\left(\mathscr{Q}, \mathscr{T}^{\prime}\right)=0$, whence $\operatorname{Hom}_{\mathscr{D} \mathscr{A}}(\tilde{\mathscr{T}}, \tilde{\mathscr{T}}[m])=0$ for $m \neq 0$, which accomplishes the proof.

2. The algebra $\tilde{\Lambda}$ can be considered as the algebra of triangular matrices

$$
\tilde{\Lambda}=\left(\begin{array}{cc}
Q & E \\
0 & \Lambda
\end{array}\right)
$$

where $Q=H^{0}(X, \mathscr{Q}), \Lambda=\operatorname{End}_{\mathscr{H}} \mathscr{T}$ and $E=\operatorname{Ext}_{\mathscr{A}_{\tilde{\mathcal{A}}}}^{1}\left(\mathscr{Q}, \mathscr{T}^{\prime}\right)$. We have already seen that there is a heredity relating chain of length $n-1$ between $\mathscr{Q}$ and $\mathscr{Q}_{1}$. On the other hand, the algebra $\Lambda$ is triangular, i.e. contains a set of orthogonal idempotents $f_{1}, f_{2}, \ldots, f_{s}$ such that $f_{i} \Lambda f_{i}$ is semi-simple (in our case equals $\mathbb{k}_{k}$ ), while $f_{i} \Lambda f_{j}=0$ if $j>i$. One easily sees that if an algebra $A$ can be presented as a matrix algebra of the form

$$
A=\left(\begin{array}{cc}
A_{1} & B \\
0 & A_{2}
\end{array}\right),
$$

where $A_{2}$ is semi-simple and $A_{1}$ is quasi-hereditary, then $A$ is also quasi-hereditary. Therefore, $\tilde{\Lambda}$ is quasi-hereditary.

3. One easily sees that $\operatorname{gl} \cdot \operatorname{dim} \Lambda \leq 2$. On the other hand, $\operatorname{gl} \operatorname{dim} Q \leq 2 n-2$ by Corollary 5.3. Then the inequality gl.dim $\tilde{\Lambda} \leq 2 n+1$ follows from (Palmer and Roos, 1973, p. 407, Corollary 4').

Thus, every rational non-commutative curve over an algebraically closed field has a categorical resolution by a finite dimensional quasi-hereditary algebra.

Recall that, for a triangulated category $\mathscr{T}$, its Rouquier dimension $\operatorname{dim} \mathscr{T}$ is defined as the smallest $d$ such that $\langle T\rangle_{d+1}=\mathscr{T}$ for some object $T$ (Rouquier, 2008). Here $\langle T\rangle_{1}$ consists of direct summands of direct sums of shifts of $T$ and $\langle T\rangle_{k+1}$ consists of direct summands of the objects $A$ such that there is an exact triangle $B \rightarrow A \rightarrow C \rightarrow B[1]$, where $B \in\langle T\rangle_{k}$ and $C \in\langle T\rangle_{1}$.

Corollary 8.6. $\operatorname{dim} \mathscr{D}^{\mathrm{c}} \mathscr{A} \leq 2 n+1$, where $\operatorname{dim}$ means the dimension of Rouquier of a triangulated category (Rouquien, 2008). Namely, $\langle\mathscr{G}\rangle_{2 n+2}=\mathscr{D}^{\mathrm{c}} \mathscr{A}$, where $\mathscr{G}=\mathscr{T} \oplus \bigoplus_{i=1}^{n} \mathscr{A}_{i} / \mathscr{A}_{n+1 . i}[-1]$.

Proof. Indeed, $\langle\tilde{\Lambda}\rangle_{2 n+2}=\mathscr{D}^{\mathrm{c}} \tilde{\Lambda}$ by (Rouquier, 2008, Proposition 7.4). As the equivalence $\mathscr{D} \tilde{\Lambda} \simeq \mathscr{D} \mathscr{\mathscr { A }}$ maps $\tilde{\Lambda}$ to $\tilde{\mathscr{T}},\langle\tilde{\mathscr{T}}\rangle_{2 n+2}=\mathscr{D}^{\mathrm{c}} \tilde{\mathscr{A}}$. Then $\langle\mathrm{DG} \tilde{\mathscr{T}}\rangle_{2 n+2}=$ $\mathscr{D}^{\mathrm{c}} \mathscr{A}$. Note that $\mathrm{G} \mathscr{M}=e_{1} \mathscr{M}$ for any $\mathscr{A}$-module $\mathscr{M}$. Therefore, $\mathrm{G} \mathscr{T}^{\prime}=\mathscr{T}$ and $\mathrm{G} \mathscr{Q}=\bigoplus_{i=1}^{n} \mathscr{A}_{i} / \mathscr{A}_{n+1, i}$. It accomplishes the proof. 
If the curve $\mathscr{A}$ is commutative, the hereditary curve $\mathscr{H}$ is regular and the algebra $\Lambda$ is hereditary (just a product of Kronecker algebras). In this case the estimate in Corollary 8.6 is $2 n$ instead of $2 n+1$. It generalizes the result of (Burban and Drozd, 2011), where the curves of level 1 were considered.

\section{ACKNOWLEDGEMENTS}

The results of this paper were mainly obtained during the stay of the second author at the Max-Plank-Institut für Mathematik. Its final version was prepared during the visit of the second and the third author to the Institute of Mathematics of the Köln University.

\section{REFERENCES}

Alonso Tarrío, L., Jeremías López, A. and Lipman J.: Local homology and cohomology on schemes. Ann. Sci. Ecole Norm. Sup. 30, 1-39 (1997)

Alonso Tarrío, L., Jeremías López, A. and Souto Salorio, M. J.: Localization in categories of complexes and unbounded resolutions. Canadian Math. J. 52, 225247 (2000)

Artin, M., Zhang, J. J.: Noncommutative projective schemes. Adv. Math. 109, 228-287 (1994)

Auslander, M., Reiten, I., Smalø S.: Representation Theory of Artin Algebras. Cambridge Univ. Press (1997)

Bourbaki, N.: Algèbre commutatif, Chapitres 5 à 7. Hermann, Paris (1975)

Bourbaki, N.: Algèbre. Chapitre X. Algèbre homologique. Springer-Verlag (1980)

Bruns, W., Herzog, J.: Cohen-Macaulay Rings. Cambridge University Press (1993)

Burban, I., Drozd, Yu.: Tilting on non-commutative rational projective curves. Math. Ann. 351, 665-709 (2011)

Burban, I., Drozd, Yu., Gavran, V.: Singular curves and quasi-hereditary algebras. arXiv:1503.04565 [math.AG]

Cartan, H., Eilenberg, S.: Homological Algebra. Princeton University Press (1956)

Cline, E., Parshall, B., Scott, L.: Finite dimensional algebras and highest weight categories. J. reine angew. Math. 391, 85-99 (1988)

Curtis, C. W., Reiner, I.: Methods of Representation Theory, vol. I. WileyInterscience (1981)

Dlab, V., Ringel. C. M.: Quasi-hereditary algebras. Illinois J. Math. 33, 280-291 (1989)

Drozd, Y. A.: Minors and theorems of reduction. Coll. Math. Soc. J. Bolyai 6, 173-176 (1973)

Drozd, Yu. A.: On existence of maximal orders. Mat. Zametki 37, 313-315 (1985)

Drozd, Yu. A., Greuel, G.-M.: Tame and wild projective curves and classification of vector bundles. J. Algebra 246, 1-54 (2001)

Gabriel, P.: Des catégories abéliennes. Bull. Soc. Math. France 90, 323-448 (1962)

Geigle, W., Lenzing, H.: A class of weighted projective curves arising in representation theory of finite dimensional algebras. Singularities, Representation of 
Algebras, and Vector Bundles. Lecture Notes Math. 1273, 265-297, SpringerVerlag, (1987)

Geigle, W., Lenzing, H.: Perpendicular categories with applications to representations and sheaves. J. Algebra 14, 273-343 (1991)

Grothendieck, A.: Éléments de géométrie algébrique: I. Publ. Math. I.H.É.S. 4 (1960)

Grothendieck, A.: Éléments de géométrie algébrique: II. Publ. Math. I.H.É.S. 8 (1961)

Hartshorne, R.: Resudues and Duality. Lecture Notes in Math. 20. Springer-Verlag (1966)

Herzog, J., Kunz, E. (ed.): Der kanonische Modul eines Cohen-Macaulay-Rings. Lecture Notes Math. 238. Springer-Verlag (1971)

König, S.: Quasi-hereditary orders. Manus. Math. 68, 417-433 (1990)

König, S.: Every order is the endomorphism ring of a projective module over a quasihereditary order. Commun. Algebra 19, 2395-2401 (1991)

Kuznetsov, A., Lunts, V. A.: Categorical resolutions of irrational singularities. arXiv:1212.6170 [math.AG].

Lang, S.: On quasi-algebraic closure. Ann. Math. 55, 373-390 (1952)

Lunts V. A., Categorical resolution of singularities, J. Algebra, 323, 2977-3003 (2010)

Miyachi J., Localization of triangulated categories and derived categories, J. Algebra, 141, 463-483 (1991)

Neeman, A.: The Grothendieck duality theorem via Bousfield's techniques and Brown representability. J. Amer. Math. Soc. 9, 205-236 (1996)

Neeman, A.: Triangulated categories. Princeton University Press (2001).

Palmer, I., Roos, J.-E.: Explicit Formulae for the Global Homological Dimensions of Trivial Extensions of Rings. J. Algebra 27, 380-413 (1973)

Reiner, I.: Maximal Orders. Clarendon Press (2003).

Ringel, C. M.: Tame Algebras and Integral Quadratic Forms. Lecture Notes in Math. 1099. Springer-Verlag (1984).

Rouquier, R.: Dimensions of triangulated categories. J. K-Theory 1, 193-256 (2008)

Spaltenstein, N.: Resolutions of unbounded complexes. Compositio Math. 65, 121154 (1988)

Van den Bergh, M.: Non-commutative crepant resolutions. The legacy of Niels Henrik Abel, 749770. Springer, (2004).

MATHEMATISCHES INSTITUt, UNIVERSitÄt ZU KÖLN, WEYERTAL 86-90, KÖLN, D-50931, GERMANY.

Institute of Mathematics, National ACADEmy of SCIEnCES of UkRaine, Tereschenkivska STR. 3, KYIV, 01601, UKRAINE.

E-mail address: burban@math.uni-koeln.de

E-mail address: y.a.drozd@gmail.com, drozd@imath.kiev.ua

E-mail address: vlgvrn@gmail.com 